\title{
Fluorine Substituted 1,2,4-Triazinones as Potential Anti-HIV-1 and CDK2 Inhibitors
}

\author{
Mohammed S. I. Makki, Reda M. Abdel-Rahman, and Khalid A. Khan \\ Department of Chemistry, Faculty of Science, King Abdulaziz University, P.O. Box 80203, Jeddah 21589, Saudi Arabia
}

Correspondence should be addressed to Khalid A. Khan; kzsakhan@hotmail.com

Received 16 February 2014; Accepted 25 April 2014; Published 21 May 2014

Academic Editor: Stojan Stavber

Copyright (C) 2014 Mohammed S. I. Makki et al. This is an open access article distributed under the Creative Commons Attribution License, which permits unrestricted use, distribution, and reproduction in any medium, provided the original work is properly cited.

\begin{abstract}
Fluorine substituted 1,2,4-triazinones have been synthesized via alkylation, amination, and/or oxidation of 6-(2-amino5-fluorophenyl)-3-thioxo-3,4-dihydro-1,2,4-triazin-5(2H)-one 1 and 4-fluoro-N-(4-fluoro-2-(5-oxo-3-thioxo-2,3,4,5-tetrahydro1,2,4-triazin-6-yl)phenyl)benzamide 5 as possible anti-HIV-1 and CDK2 inhibitors. Alkylation on positions 2 and 4 in 1,2,4triazinone gave compounds 6-8. Further modification was performed by selective alkylation and amination on position 3 to form compounds 9-15. However oxidation of 5 yielded compounds 16-18. Structures of the target compounds have been established by spectral analysis data. Five compounds $(\mathbf{5}, \mathbf{1 1}, \mathbf{1 4}, \mathbf{1 6}$, and 17) have shown very good anti-HIV activity in MT-4 cells. Similarly, five compounds (1, 3, and 14-16) have exhibited very significant CDK2 inhibition activity. Compounds 14 and 16 were found to have dual anti-HIV and anticancer activities.
\end{abstract}

\section{Introduction}

Human immunodeficiency virus (HIV) type-1 is the causative agent of acquired immunodeficiency syndrome (AIDS), which is one of the serious global health problems [1]. The currently approved anti-HIV drugs can be divided into five groups: reverse transcriptase inhibitors (RTIs), protease inhibitors [2] (PIs), fusion inhibitors (FIs), coreceptor inhibitors (CRIs), and integrase inhibitors (INIs). This arsenal of drugs, which is used in combinations, has moved the prognosis of HIV patients from that of high morbidity and mortality to, for many at least, a chronic, manageable but still complex disease [3-5]. However, the use of these drugs has been relatively limited by their toxicity [6], drug resistance development [7], and, more worryingly, the fact that some newly HIV-infected patients carry viruses that are already resistant to the currently approved AIDS treatments [8]. These issues along with drug-related side effects make it apparent that new anti-HIV drugs with novel mechanisms of action are clearly needed.

On the other hand, during the past 30 years, a variety of approaches have been taken for cancer chemotherapy and many antitumor drugs have been developed for clinical use. In the treatment of solid tumors, however, the conventional approaches have met with only limited success and cancer still remains as one of the leading causes of human mortality [9].

Chemotherapy drugs are sometimes feared because of a patients' concern about its toxic effects. There are three goals associated with the use of the most commonly used anticancer agents: (a) damage the DNA of the affected cancer cells, (b) inhibit the synthesis of new DNA strands to stop the cells from replicating, and (c) stop the mitosis or the actual splitting of the original cell into two new daughter cell [10].

In the past few years fluorine substituted heterocyclic nitrogen systems have been incorporated into drug discovery research [11-23] to improve the physicochemical properties of drugs. Organic fluorine is a prominent tool in the design and improvement of pharmacokinetic properties of drug molecules. Replacing hydrogen and other functional groups with fluorine can have a dramatic effect on the modulation of electronic, lipophilic, and steric parameters, all of which can critically influence both the pharmacodynamic and pharmacokinetic properties of drugs. Substitution of fluorine into a potential drug molecule not only alters the electronic environment, but it also influences the properties of neighboring functional groups. Fluorine can have significant effects on the binding affinity in protein-ligand complexes. The DNA polymerase inhibitors fludarabine (I) (F-ara-A), 
<smiles>Nc1nc(F)nc2c1ncn2C1OC2OC1C(COP(=O)(O)O)C2O</smiles>

(I) Fludarabine

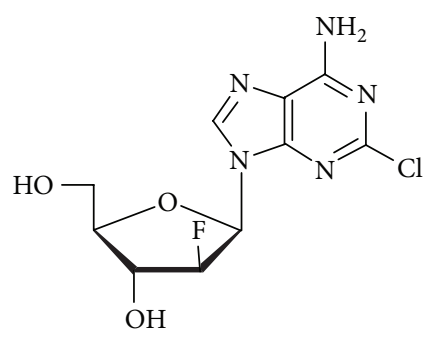

(II) Chlofarabine<smiles>Nc1ccn(C2O[C@H](CO)[C@@H](O)C2=CF)c(=O)n1</smiles>

(III) Tezacitabine<smiles>Cc1ccc(NC(=O)c2ccc(CN3CCN(C)CC3)cc2)cc1Nc1nccc(-c2cccnc2)n1</smiles>

(IV) Gleevec<smiles>CN1CCC(Nc2ncc3ncnc(Cc4ccc(F)c(Cl)c4)c3n2)CC1</smiles>

(V) BX-1382BS<smiles>CC(C)(N)c1ccc(-c2nc(Nc3ccc(CCN4CCOCC4)cc3)ncc2C#N)cc1</smiles>

(VI) JNJ-17029259

Figure 1

clofarabine (II), and tezacitabine (III) are used as cancer chemotherapeutic agents [24], while Gleevec (IV) is used as catalytic inhibitor of imatinib mesylate [25]. In addition, BX1382BS (V) showed a significant activity as protein kinase inhibitor [26] and a cyanopyrimidine scaffold JNJ-17029259 (VI) is an oral inhibitor of VEGF-mediated signal transduction [27] (Figure 1). Recently a great deal of synthetic efforts has been spent on fluorinated uncondensed 1,2,4-triazines by our group searching for new anti-HIV and anticancer agents [28-38] (compounds A-F; Figure 2).

Fluorine incorporation on key positions plays a significant role to alter the physicochemical and biological characteristics of organic compounds. Frequently, it is found that a fluorine substituent leads to an enhancement of the binding affinity of a molecule with proteins through a noncovalent bond formation. Fluorine increases binding affinity, reduces plasma protein binding leading to a higher free fraction of the drug, and increases cell penetration. The combination of these effects results in a dramatically improved biological activity. Based on these valuable observations and in part of our continuing efforts in drug development, the present work describes an attempt towards the synthesis of fluorine substituted 1,2,4-triazinones. The purpose of the work is to extend the scope of our previous studies $[29,30]$ and to substitute fluorine on various positions in 1,2,4-triazines in order to obtain effective anti HIV-1 and CDK2 inhibiting agents.

\section{Experimental}

2.1. Chemistry. Melting points were determined on an electrothermal Bibby Stuart Scientific melting point apparatus and are uncorrected. The infrared (IR) spectra were recorded on PerkinElmer RXI FT-IR infrared spectrophotometer using the $\mathrm{KBr}$ pellet technique. Electronic absorption spectra were recorded in DMF on Shimadzu UV-Visible 3101 PC spectrophotometer. ${ }^{1} \mathrm{H}$ and ${ }^{13} \mathrm{C}$ NMR spectra were recorded on a Bruker DPX-400 FT NMR spectrometer using tetramethylsilane as the internal standard DMSO- $d_{6}$ as a solvent (chemical shifts in $\delta, \mathrm{ppm}) .{ }^{19} \mathrm{~F}$ NMR spectra were determined at $84.25 \mathrm{MHz}$ using hexafluorobenzene as an internal standard. Splitting patterns were designated as follows: $s$ : singlet; $m$ : multiplet. Mass spectra were measured on a GCMS-Q 1000 Ex spectrometer. Elemental analyses were performed on a 2400 PerkinElmer Series 2 analyzer. Follow-up of the reactions and checking the homogeneity of the compounds were made by TLC on silica gel-protected aluminum sheets (Type 60 F254, Merck) and the spots were detected by exposure to UV-lamp at $\lambda 254$.

The anti-HIV and anticancer activity were evaluated in DTP, DCT, National Cancer Institute, Bethesda, MD 20892, USA.

2.1.1. 6-(2-Amino-5-fluorophenyl)-3-thioxo-3,4-dihydro-1,2,4triazin-5(2H)-one (1). A mixture of 5-fluoroisatin $(0.001 \mathrm{~mol}$ in warm $5 \%$ aqueous $\mathrm{NaOH}$ ) and thiosemicarbazide $(0.001 \mathrm{~mol}$, in hot water $10 \mathrm{~mL})$ was refluxed for $2 \mathrm{~h}$. The reaction mixture was then poured onto ice and neutralized with dil. HCl. The solid thus obtained was filtered off and crystallized from ethanol to give $\mathbf{1}$ as pale yellow crystals. Yield $80 \%$, m.p. $285-287^{\circ} \mathrm{C}$. UV: $\lambda_{\max }(\varepsilon): 282.8(2.06) \mathrm{nm}$. IR $\left(\nu, \mathrm{cm}^{-1}\right): 3528,3300\left(\mathrm{NH}, \mathrm{NH}_{2}\right), 1661(\mathrm{C}=\mathrm{O}), 1385(\mathrm{NCSN})$, 1255 (C-F), 865 (aryl C-H), 658 (C-F). ${ }^{1} \mathrm{H}$ NMR ( $\delta$, ppm): 
<smiles>O=C(Nc1ccccc1-c1n[nH]c(=S)[nH]c1=O)C(F)(F)F</smiles>

(a)<smiles>FC(F)(F)C(F)(F)n1c2ccccc2c2nnc(S)nc21</smiles>

(d)<smiles>[R]Cn1nc(-c2ccccc2NC(=O)C(F)(F)F)c(=O)n(C[R])c1=S</smiles>

(b)<smiles>NNc1nnc(-c2ccccc2NC(=O)C(F)(F)F)c(=O)[nH]1</smiles>

(e)<smiles>O=C([O-])Nc1ccccc1-c1nnc(SCC(=O)Nc2ccccc2)[nH]c1=O</smiles>

(c)<smiles>O=C(Nc1ccccc1-c1nnc(NNc2ccccc2)[nH]c1=O)C(F)(F)F</smiles>

(f)

FIGURE 2

13.47, (s, 1H, NH), 7.52-7.42, 6.93-6.87 (3H, aromatic $\mathrm{H})$, $3.2\left(\mathrm{~s}, 2 \mathrm{H}, \mathrm{NH}_{2}\right) .{ }^{13} \mathrm{C} \mathrm{NMR}(\delta, \mathrm{ppm}): 172.74(\mathrm{C}=\mathrm{S}), 156-152$ $(\mathrm{C}=\mathrm{O}), 144.53(\mathrm{C}-\mathrm{F}), 138.73(\mathrm{C}=\mathrm{N}), 119.68,117.60,117.45$, $116.50,116.30$ (aromatic carbons), 77.27-76.84, 39.91-39.07 (C-C, C-N carbons). MS (relative intensity): $238\left(\mathrm{M}+\mathrm{H}_{2} \mathrm{O}\right.$, 254, 5\%), 206 (207, 36), 182 (10), 108 (1.1), 103 (100), 75 (35). Anal. calcd. $\mathrm{C}_{9} \mathrm{H}_{7} \mathrm{~N}_{4} \mathrm{SOF}(238)$ : C, 45.38; H, 2.93; N, 23.52; S, 13.0; F, 7.98. Found: C, 44.88; H, 2.55; N, 23.41; S, 12.85; F, 7.77. ${ }^{19}$ F NMR: $\delta-127$ ppm.

2.1.2. 2-((6-(2-((Carboxymethyl)amino)-5-fluorophenyl)-5oxo-4,5-dihydro-1,2,4-triazin-3-yl)thio)acetic acid (2). A mixture of $1(0.001 \mathrm{~mol})$ and monochloroacetic acid $(0.002 \mathrm{~mol})$ in ethanolic $\mathrm{NaOH}(5 \%, 50 \mathrm{~mL})$ was warmed for $30 \mathrm{~min}$ and then cooled and poured onto ice-cold dil. acetic acid. The product is precipitated as pale solid which was then filtered and crystallized from acetic acid to give yellow crystals of compound 2 . Yield 70\%, m.p. $262-264^{\circ} \mathrm{C}$. UV: $\lambda_{\max }(\varepsilon): 283.3(1.93) \mathrm{nm}$. IR $\left(\nu, \mathrm{cm}^{-1}\right): 3531(\mathrm{OH})$, $3300(\mathrm{NH}), 2929,2861\left(2 \mathrm{CH}_{2}\right), 1660(\mathrm{C}=\mathrm{O}), 1499,1439$ (deformation $\mathrm{CH}_{2}$ ), 1385 (NCSN), $1255(\mathrm{C}-\mathrm{F}), 865$ (aryl C-H), 658 (C-F). ${ }^{1} \mathrm{H}$ NMR ( $\delta$, ppm): 13.38, (s, 1H, OH), 11.82 (s, NH), 7.55-7.37, 6.85-6.71 (aromatic H), 3.55-3.54, 3.53-3.51 (each s, $\left.2 \mathrm{CH}_{2}\right) .{ }^{13} \mathrm{C} \mathrm{NMR}(\delta$, ppm): $172.67,152.42(2 \mathrm{C}=\mathrm{O})$, 145 (C-F), 117.64, 117.50, 116.41, (aromatic carbons), 77.15, 76.94, 76.73 39.91-39.07 (C-S, C-O, and C-N carbons), 39.91, $39.07\left(2 \mathrm{CH}_{2}\right)$. MS (relative intensity): $266\left(\mathrm{M}-2\left(\mathrm{CO}_{2}\right), 100\right.$. Anal. calcd. $\mathrm{C}_{13} \mathrm{H}_{11} \mathrm{~N}_{4} \mathrm{SO}_{5} \mathrm{~F}$ (354): C, 44.07; H, 3.10; N, 16.0; S, 9.03; F, 5.35. Found: C, 43.78; H, 2.85; N, 15.69; S, 8.81; F, 5.21. ${ }^{19} \mathrm{~F}$ NMR: $\delta-130 \mathrm{ppm}$.

\subsubsection{6-(5-Fluoro-2-(methylamino)phenyl)-3-(methylthio)- 1,2,4-triazin-5(4H)-one (3)}

Procedure A. A mixture of compound $2(0.050 \mathrm{~mol})$ and $10 \%$ $\mathrm{K}_{2} \mathrm{CO}_{3}(20 \mathrm{~mL})$ was refluxed for $10 \mathrm{~min}$ and then left to acquire room temperature. The reaction mixture was now poured in an ice-cold dil. $\mathrm{HCl}$. The precipitated solid was filtered and crystallized as faint yellow crystals. Yield 50\%, m.p. $163-164^{\circ} \mathrm{C}$.

Procedure B. A mixture of compound 1 (0.001), methyl iodide $(0.002 \mathrm{~mol})$, and $1 \%$ alcoholic $\mathrm{KOH}(20 \mathrm{~mL})$ was stirred at room temperature for $4 \mathrm{~h}$. The reaction mixture was then neutralized with dil. $\mathrm{HCl}$. The resulting light brown solid was filtered and crystallized from ethanol to form yellowish crystals of compound 3 . Yield $65 \%$, m.p. $162-163^{\circ} \mathrm{C}$. UV: $\lambda_{\max }$ $(\varepsilon): 315.4(1.154) \mathrm{nm}$. IR $\left(\nu, \mathrm{cm}^{-1}\right): 3310(\mathrm{NH}), 2929,2862$ $\left(2 \mathrm{CH}_{2}\right), 1661(\mathrm{C}=\mathrm{O}), 1499,1438$ (deformation $\left.\mathrm{CH}_{2}\right), 1385$ (NCSN), $1255(\mathrm{C}-\mathrm{F}), 865(\operatorname{aryl} \mathrm{C}-\mathrm{H}), 658(\mathrm{C}-\mathrm{F})$. MS (relative intensity): $266\left(\mathrm{M}+\mathrm{H}_{2} \mathrm{O}, 284,5 \%\right), 110$ (5.5), 104 (100), 95 (1.18), 88 (100), 47 (65.0). Anal. calcd. $\mathrm{C}_{11} \mathrm{H}_{11} \mathrm{~N}_{4} \mathrm{SOF}$ (266): C, 49.95; H, 4.13; N, 21.05; S, 12.03; F, 7.14. Found: C, 49.71; H, 4.01; N, 20.99; S, 11.88; F, 6.91. ${ }^{19}$ F NMR: $\delta-126$ ppm.

2.1.4. $2,2^{\prime}-\left(\left(\left(3,3^{\prime}-((\right.\right.\right.$ Carboxymethylene $) b i s($ sulfanediyl $)) b i s(5-$ oxo-4,5-dihydro-1,2,4-triazine-6,3-diyl)) bis(4-fluoro-2,1phenylene))bis(azanylylidene))diacetic acid (4). A mixture of compound $1(0.001 \mathrm{~mol})$ and 1,1-dichloroacetic acid $(0.003 \mathrm{~mol})$ in DMF $(20 \mathrm{~mL})$ was refluxed for $10 \mathrm{~min}$, cooled, and then poured on ice. The solid thus separated was filtered and crystallized from THF to give deep orange crystals. Yield $66 \%$, m.p. $280-282^{\circ} \mathrm{C}$. UV: $\lambda_{\max }(\varepsilon): 266.5(1.51) \mathrm{nm}$. IR $(\nu$, $\left.\mathrm{cm}^{-1}\right)$ : 3534, $3480(\mathrm{OH}), 3350(\mathrm{NH}), 2929,2861$ (aliphatic $\mathrm{C}-\mathrm{H}), 1680,1660(\mathrm{C}=\mathrm{O}), 1499,1439$ (deformation $\mathrm{C}-\mathrm{H})$, 1386 (NCSN), 1255 (C-F), 865 (aryl C-H), 658 (C-F). MS (relative intensity): 644 (M-208, 3\%), 298 (100), 167 (31.01), 149 (100), 85 (5.00). Anal. calcd. $\mathrm{C}_{24} \mathrm{H}_{14} \mathrm{~N}_{8} \mathrm{~S}_{2} \mathrm{O}_{8} \mathrm{~F}_{2}$ (266): C, 44.72; H, 2.17; N, 17.36; S, 9.9; F, 5.95. Found: C, 44.51; H, 2.11; N, 17.12; S, 9.55; F, 5.71. ${ }^{19}$ F NMR: $\delta-128$ ppm.

2.1.5. 4-Fluoro-N-(4-fluoro-2-(5-oxo-3-thioxo-2,3,4,5-tetrahydro-1,2,4-triazin-6-yl)phenyl)benzamide (5). A mixture of compound $1 \quad(0.001 \mathrm{~mol})$ and 4-fluorobenzoyl chloride 
$(0.001 \mathrm{~mol})$ in dry pyridine $(10 \mathrm{~mL})$ was warmed for $10 \mathrm{~min}$, cooled, and then poured on ice. The precipitated solid was filtered and crystallized from dioxane to give faint yellow crystals of 5 . Yield $85 \%$, m.p. $238-240^{\circ} \mathrm{C}$. UV: $\lambda_{\max }$ $(\varepsilon): 288.4(1.28) \mathrm{nm}$. IR $\left(\nu, \mathrm{cm}^{-1}\right): 3370(\mathrm{NH}), 3320(\mathrm{NH})$, 1663, 1610 (C=O, CONH), 1385 (NCSN), 1255 (C-F), 1190 (C-S), 864 (aryl C-H), $658(\mathrm{C}-\mathrm{F}) .{ }^{1} \mathrm{H}$ NMR $(\delta, \mathrm{ppm}): 13.75$, $(\mathrm{NH}), 10.07(\mathrm{NH}), 8.60(\mathrm{NH}), 7.98-7.82,7.53-7.41,7.10-7.05$ (aromatic H). ${ }^{13} \mathrm{C}$ NMR $(\delta, \mathrm{ppm}): 173.15(\mathrm{C}=\mathrm{S}), 164.97-$ 163.30, 158.87-158.57 (C=O), $146.91(\mathrm{C}-\mathrm{F}), 144.70(\mathrm{C}-\mathrm{F})$, $138.07(\mathrm{C}-\mathrm{N}), 132.21,130.16,129.57,129.51,125.5,124.26$ (aromatic carbons), 116.63, 115.04, 114.90 (C-S, C-O, C-N) 77.50-77.07, 40.03, 39.08. MS (relative intensity): $360(\mathrm{M}+4$, 6\%), 265 (78.0), 123 (12.05), 96 (2.0), 89 (100), 76 (75.0). Anal. calcd. $\mathrm{C}_{16} \mathrm{H}_{10} \mathrm{~N}_{4} \mathrm{SO}_{2} \mathrm{~F}_{2}$ (360): C, 53.30; H, 2.78; N, 15.50; S, 9.16; F, 10.55. Found: C, 52.88; H, 2.55; N, 15.02; S, 8.95; F, 10.35. ${ }^{19}$ F NMR: $\delta-126$ and -129 ppm.

2.1.6. N-(2-(2,4-Disubstituted-5-oxo-3-thioxo-2,3,4,5-tetrahydro-1,2,4-triazin-6-yl)-4-fluorophenyl)-4-fluorobenzamide (6-8). A mixture of $5(0.001 \mathrm{~mol})$ methanol or morpholine or $2,2^{\prime}$-dipyridylamine $(0.001 \mathrm{~mol})$ in methanol $(20 \mathrm{~mL})$ and formaldehyde $(0.001 \mathrm{~mol})$ was refluxed for $2 \mathrm{~h}$. The reaction mixture was cooled to acquire room temperature and then poured onto ice-cold water. The solid product precipitated was filtered and crystallized from methanol to form yellow-orange crystals.

6. Yield $72 \%$, m.p. $310-312^{\circ} \mathrm{C}$. IR $\left(\nu, \mathrm{cm}^{-1}\right): 3527(\mathrm{OH}), 3100$ (NH), 2935, 2890 (aliphatic C-H), 1660, 1600 (C=O, CONH), 1498, 1440 (deformation $\mathrm{CH}_{2}$ ), 1386 (NCSN), 1255 (C-F), 864 (aryl C-H), $658(\mathrm{C}-\mathrm{F}) .{ }^{1} \mathrm{H}$ NMR $(\delta, \mathrm{ppm}): 12.54$ (s, $1 \mathrm{H}$, $\mathrm{NH}$ ), 10.15, 8.04 (Two s, $2 \mathrm{OH}$ ), 7.96-7.94, 7.87-7.83, 7.647.63 (aromatic $\mathrm{H}$ ), 2.49 and 2.48 (Two s, $2 \mathrm{CH}_{2}$ ). ${ }^{13} \mathrm{C} \mathrm{NMR}$ $(\delta$, ppm): $164.99(\mathrm{C}=\mathrm{S}), 163.32,157.02(\mathrm{C}=\mathrm{O}), 135.99(\mathrm{C}-$ F), 130.39, 130.20, 129.53, 129.44, 129.36, 123.45, (aromatic carbons), 115.14, 114.99 (C-S, C-O) 77.38-76.95, 39.83, 39.29. Anal. calcd. $\mathrm{C}_{18} \mathrm{H}_{14} \mathrm{~N}_{4} \mathrm{SO}_{4} \mathrm{~F}_{2}$ (420): C, 51.42; H, 3.36; N, 13.30; S, 7.61; F, 9.04. Found: C, 51.23; H, 3.01; N, 13.11; S, 7.51; F, 8.88. ${ }^{19}$ F NMR: $\delta-126$ and -129 ppm.

7. Yield 70\%, m.p. $260-262^{\circ} \mathrm{C}$. Anal. calcd. $\mathrm{C}_{26} \mathrm{H}_{28} \mathrm{~N}_{6} \mathrm{SO}_{4} \mathrm{~F}_{2}$ (558): C, 55.90; H, 5.02; N, 15.05; S, 5.70; F, 6.81. Found: C, $55.68 ; \mathrm{H}, 4.98 ; \mathrm{N}, 14.79 ; \mathrm{S}, 5.35 ; \mathrm{F}, 6.66$.

8. Yield 72\%; m.p. $202-204^{\circ} \mathrm{C}$. Anal. calcd. $\mathrm{C}_{38} \mathrm{H}_{28} \mathrm{~N}_{10} \mathrm{SO}_{2} \mathrm{~F}_{2}$ (726): C, 62.79; H, 3.88; N, 19.28; S, 4.27; F, 5.23. Found: C, 62.33; H, 3.58; N, 19.11; S, 4.15; F, 4.89.

2.1.7. 4-Fluoro-N-(4-fluoro-2-(3-(substituted-amino)-5-oxo4,5-dihydro-1,2,4-triazin-6-yl)phenyl)benzamide (9-12). A mixture of $5(0.001 \mathrm{~mol})$ and sulfathiazole or sulfaoxazole or 4-fluoroaniline or $2,2^{\prime}$-dipyridylamine $(0.001 \mathrm{~mol})$ in absolute ethanol $(50 \mathrm{~mL})$ was refluxed for $6 \mathrm{~h}$. On cooling a solid is separated which is filtered and crystallized from ethanol to give yellow crystals of 9-12.

9. Yield $85 \%$, m.p. $172-174^{\circ} \mathrm{C}$. IR $\left(\nu, \mathrm{cm}^{-1}\right): 3484-3310(\mathrm{NH})$, 1658, 1610 (C=O, CONH), 1386 (NCSN), 1255 (C-F), 864 (aryl C-H), $658(\mathrm{C}-\mathrm{F}) .{ }^{1} \mathrm{H}$ NMR $(\delta, \mathrm{ppm}): 13.72,10.06,8.00$ (each s, $3 \mathrm{NH}$ ), 7.94 (s, 1H, $\mathrm{NHSO}_{2}$ ), 7.89, 7.84 (each s, 2H, thiazole), 7.56, 7.49, 7.39, 7.38, 7.10, 7.09, 7.78, 7.07, 6.79, 6.78, 6.55, 6.53, 6.36 (aromatic H). ${ }^{13} \mathrm{C}$ NMR $(\delta, \mathrm{ppm}): 173.18(\mathrm{C}-$ $\mathrm{SO}_{2}$ ), 167.90, 157.28 (C=O), 144.81 (C-F), 132.24, $132.22(\mathrm{C}-$ $\mathrm{N}), 130.22$, 130.21, 129.62, 129.56, 128.93, 127.50, 125.58, 125.53, $124.40,123.14,116.78,116.73,116.62,116.58,115.03,114.88$, 112.82, 106.46 (aromatic carbons), 77.62-77.19. Anal. calcd. $\mathrm{C}_{25} \mathrm{H}_{17} \mathrm{~N}_{7} \mathrm{~S}_{2} \mathrm{O}_{4} \mathrm{~F}_{2}$ (581): C, 51.63; H, 2.92; N, 16.86; S, 11.01; F, 6.55. Found: C, 51.34; H, 2.85; N, 16.70; S, 10.88; F, 6.35.

10. Yield $82 \%$, m.p. $178-180^{\circ} \mathrm{C}$. Anal. calcd. $\mathrm{C}_{25} \mathrm{H}_{17} \mathrm{~N}_{7} \mathrm{SO}_{5} \mathrm{~F}_{2}$ (565): C, 53.09; H, 3.00; N, 17.16; S, 5.66; F, 6.72. Found: C, 52.88; H, 2.89; N, 17.01; S, 5.41; F, 6.55 .

11. Yield 89\%, m.p. $247-249^{\circ}$ C. UV: $\lambda_{\max }(\varepsilon): 288.7(1.09) \mathrm{nm}$. IR $\left(\nu, \mathrm{cm}^{-1}\right): 3500,3310,3290(\mathrm{NH}), 1661,1620(\mathrm{C}=\mathrm{O}), 1385$ (NCSN), 1255 (C-F), 865 (aryl C-H), 658 (C-F). ${ }^{1} \mathrm{H}$ NMR ( $\delta$, ppm): 13.79, 11.72, 10.92 (each s, $3 \mathrm{NH}$ ), 7.94, 7.88, 7.86, $7.70,7.54,7.53,7.47,7.25,7.09,7.07,7.06$ (aromatic H). ${ }^{13} \mathrm{C} \mathrm{NMR}$ $(\delta, \mathrm{ppm}):$ 164.95-163.27, 158.86-157.24 (C=O), 146.34, 144.74, 138.74 (C-F), 132.22 (C-N), 129.56, 129.51, 125.48, 124.46, $116.77,116.61,115.02,114.88$ (aromatic carbons), 77.49-77.07. MS (relative intensity): 437 (M-45, 392, 1.0\%), 166 (32.0), 148 (100), 122 (1.1), 110 (11), 95 (3.0). Anal. calcd. $\mathrm{C}_{22} \mathrm{H}_{14} \mathrm{~N}_{5} \mathrm{~S}_{2} \mathrm{~F}_{3}$ (437): C, 60.41; H, 3.20; N, 16.01; F, 13.04. Found: C, 59.59; H, 3.10; N, 15.66; F, $12.85 .{ }^{19}$ F NMR: $\delta-127$ and -129 ppm.

12. Yield $80 \%$, m.p. $200-202^{\circ}$ C. UV: $\lambda_{\text {max }}(\varepsilon): 271.8(1.51) \mathrm{nm}$. IR $\left(\nu, \mathrm{cm}^{-1}\right)$ : 3525-3100 (3 NH), 1659, $1610(\mathrm{C}=\mathrm{O}, \mathrm{CONH})$, 1386 (NCSN), 1255 (C-F), 865 (aryl C-H), $658(\mathrm{C}-\mathrm{F}) .{ }^{1} \mathrm{H}$ $\operatorname{NMR}(\delta, \mathrm{ppm}): 13.79,11.72,10.92$ (each s, $3 \mathrm{NH}), 9.98-9.74$, $8.16-8.15,7.96-7.95,7.94$ (4H of pyridine), 7.86-7.84, 7.59-7.55, 7.40-7.37, 7.10-7.08, 7.07-7.05, 6.82-6.81, 6.80-6.79 (aromatic H). ${ }^{13} \mathrm{C}$ NMR $(\delta, \mathrm{ppm}): 164.92-163.25,158.87-157.25(\mathrm{C}=\mathrm{O})$, $145.44,144.84(\mathrm{C}-\mathrm{F}), 137.71(\mathrm{C}-\mathrm{N}), 132.24,130.23,129.61$, $129.55,125.58,125.53,124.45,124.39$ (aromatic carbons), $116.76,116.69,116.54,115.69,114.99$ (pyridine carbons). Anal. calcd. $\mathrm{C}_{26} \mathrm{H}_{17} \mathrm{~N}_{7} \mathrm{O}_{2} \mathrm{~F}_{2}$ (497): C, 62.75; H, 3.40; N, 19.71; F, 7.62. Found: C, 62.45; H, 3.30; N, 19.55; F, 7.43.

\subsubsection{4-Fluoro-N-(4-fluoro-2-(3-((4-fluorophenyl)amino)-5- oxo-4,5-dihydro-1,2,4-triazin-6-yl)phenyl)benzamide}

(11)

Procedure A. Equimolar mixture of compound 14 and 4fluoroaniline was refluxed for $6 \mathrm{~h}$ then cooled. The solid thus obtained was filtered and crystallized from ethanol to give $\mathbf{1 1}$ as faint yellow crystals. Yield 78\%, m.p. $247-249^{\circ} \mathrm{C}$.

Procedure B. An equimolar mixture of compound 5 and 4fluoroaniline in ethanol $(50 \mathrm{~mL})$ was refluxed for $6 \mathrm{~h}$ and then cooled. The resulting solid was filtered and crystallized from ethanol to give faint yellow crystals of 11 . Yield 75\%, m.p. 247$249^{\circ} \mathrm{C}$.

2.1.9. 2-((6-(5-Fluoro-2-(4-fluorobenzamido)phenyl)-5-oxo4,5-dihydro-1,2,4-triazin-3-yl)thio)acetic acid (13). A mixture 
of $5(0.001 \mathrm{~mol})$ and monochloroacetic acid $(0.001 \mathrm{~mol})$ in pyridine $(20 \mathrm{~mL})$ was refluxed for $15 \mathrm{~min}$, cooled, and then poured onto ice-cold dil. $\mathrm{HCl}$. The resulting solid was filtered and crystallized from ethanol to give $\mathbf{1 3}$ as yellow crystals. Yield $75 \%$, m.p. $190-192^{\circ} \mathrm{C}$. IR $\left(\nu, \mathrm{cm}^{-1}\right)$ : $3550-3100$ (br, OH and $\mathrm{NH}), 2919,2885(\mathrm{C}-\mathrm{H}), 1658,1610(\mathrm{C}=\mathrm{O}, \mathrm{CONH}), 1489$, 1429 (deformation $\left.\mathrm{CH}_{2}\right), 1386(\mathrm{NCSN}), 1255(\mathrm{C}-\mathrm{F}), 865$ (aryl C-H), $658(\mathrm{C}-\mathrm{F}) .{ }^{1} \mathrm{H}$ NMR $(\delta, \mathrm{ppm}): 14.45(\mathrm{NH}), 12.58$ $(\mathrm{NH}), \quad 10.25-10.08 \quad(\mathrm{OH}), \quad 7.88-7.85, \quad 7.84-7.83, \quad 7.65-7.31$, 7.15-7.14, 7.13-7.12, 7.10-7.07 (aromatic H), $3.96\left(\mathrm{CH}_{2}\right) \cdot{ }^{13} \mathrm{C}$ NMR ( $\delta$, ppm): $168.74(\mathrm{C}=\mathrm{O}), 164.99(\mathrm{C}=\mathrm{O}), 159.27-157.65$ $(\mathrm{C}=\mathrm{O}), 132.87-132.85$ (C-S), 130.20, 130.18, 129.61, 129.49, $129.43,127.19,126.20,117.18,117.01,116.73,116.58,115.17,115.03$, (aromatic carbons), 77.89-77.45, 40.88, $31.84\left(\mathrm{CH}_{2}\right) . \mathrm{MS}$ (relative intensity): 418 (M-28, 390, 1.0\%), 391 (1.5), 149 (100), 132 (2.8), 91 (1.10), 57 (47.00). Anal. calcd. $\mathrm{C}_{18} \mathrm{H}_{12} \mathrm{~N}_{4} \mathrm{SO}_{4} \mathrm{~F}_{2}$ (418): C, 53.11; H, 2.87; N, 13.35; S, 7.65; F, 9.09. Found: C, 52.88; H, 2.58; N, 13.05; S, 7.33; F, 8.79.

\subsubsection{4-Fluoro-N-(4-fluoro-2-(3-(methylthio)-5-oxo-4,5- dihydro-1,2,4-triazin-6-yl)phenyl)benzamide (14)}

Procedure A. A mixture of compound $13(0.050 \mathrm{~mol})$ and 10\% ethanolic $\mathrm{K}_{2} \mathrm{CO}_{3}(10 \mathrm{~mL})$ was warmed for $15 \mathrm{~min}$, cooled, and then poured onto ice-cold dil. $\mathrm{HCl}$. The solid product was filtered and crystallized from dioxane to give deep orange crystals of 14 . Yield $82 \%$, m.p. $228-230^{\circ} \mathrm{C}$.

Procedure B. A mixture of compound $5(0.001 \mathrm{~mol})$ and $\mathrm{CH} 3 \mathrm{I}$ $(0.001 \mathrm{~mol})$ in ethanolic $\mathrm{KOH}(1 \%, 20 \mathrm{~mL})$ was stirred at room temperature for $12 \mathrm{~h}$. The resulting reaction mixture was acidified with dil. $\mathrm{HCl}$. A brownish orange precipitate was separated which on crystallization with dioxane gave 14 as orange crystals. Yield $80 \%$, m.p. 228-229॰C. Mixed m.p. 228。C. IR ( $\nu, \mathrm{cm}-1): 3484-3250$ (br, NH, NH), 2930, 2864 (C$\mathrm{H}), 1657,1620(\mathrm{C}=\mathrm{O}, \mathrm{CONH}), 1489,1449$ (deformation $\mathrm{CH} 2$ ), 1386 (NCSN), 1255 (C-F), 864 (aryl C-H), 658 (C-F). 1H $\operatorname{NMR}(\delta, \mathrm{ppm}): 12.65,12.37-12.35$ (each s, NH, NH), 7.89-7.88, 7.87, 7.40-7.25, 7.14-7.13, 7.12, 7.12-7.10, 7.08-7.04 (aromatic H). 13C NMR $(\delta, \mathrm{ppm}): 163.60,159.38(\mathrm{C}=\mathrm{O}), 157.79,147.45(\mathrm{C}-$ F), 132.89 (C-S), 129.85, 129.28, 129.26, 129.22, 129.20, 127.43, $126.28,126.25,117.19,117.03,116.85,116.82,116.70,116.67,115.14$, $115.12,115.00,114.97$ (aromatic and heteroaromatic carbons), 77.21-76.78, 39.91-39.07 (CH3). Anal. calcd. C17H12N4SO2F2 (374): C, 54.50; H, 3.20; N, 14.89; S, 8.56; F, 10.17. Found: C, 53.89; H, 3.01; N, 14.55; S, 8.19; F, 10.11.

2.1.11. 6-(2-Amino-5-fluorophenyl)-3-((4-fluorophenyl)amino)1,2,4-triazin-5(4H)-one (15). A mixture of $1(0.001 \mathrm{~mol})$ and 4-fluoroaniline $(0.001 \mathrm{~mol})$ in ethanol $(50 \mathrm{~mL})$ was refluxed for $6 \mathrm{~h}$. On cooling a solid was precipitated which was filtered and crystallized from ethanol to form yellowish crystals of $\mathbf{1 5}$. Yield $80 \%$, m.p. $308-310^{\circ} \mathrm{C}$. IR $\left(\nu, \mathrm{cm}^{-1}\right): 3534,3300\left(\mathrm{NH}_{2}\right.$, $\mathrm{NH}), 1661(\mathrm{C}=\mathrm{O}), 1386(\mathrm{NCSN}), 1255(\mathrm{C}-\mathrm{F}), 864(\operatorname{aryl~C}-\mathrm{H})$, 658 (C-F). Anal. calcd. $\mathrm{C}_{15} \mathrm{H}_{11} \mathrm{~N}_{5} \mathrm{OF}_{2}$ (315): C, 58.00; H, 3.49; $\mathrm{N}, 22.22 ; \mathrm{F}, 12.06$. Found: C, 57.72; H, 3.15; N, 21.88; F, $11.78 .{ }^{19} \mathrm{~F}$ NMR: $\delta-127$ and -129 ppm.
2.1.12. Aroylation of $\mathbf{1 5}$ to Form 11. An equimolar mixture of 15 and 4-fluorobenzoyl chloride in DMF $(20 \mathrm{~mL})$ was warmed for $10 \mathrm{~min}$, cooled, and then poured onto ice. The resultant solid was filtered and crystallized from ethanol to give 11 as yellowish crystals. Yields $82 \%$, m.p. $248^{\circ} \mathrm{C}$. Mixed m.p. $247^{\circ} \mathrm{C}$.

2.1.13. $\quad N, N^{\prime}-\left(\left(3,3^{\prime}\right.\right.$-Disulfanediylbis(5-oxo-4,5-dihydro-1,2,4triazine-6,3-diyl)) bis(4-fluoro-2,1-phenylene))bis(4-fluorobenzamide) (16). A mixture of compound $5(0.002 \mathrm{~mol})$ and sulfur $(0.002 \mathrm{~mol})$ flowers in dry benzene $(100 \mathrm{~mL})$ was refluxed for $4 \mathrm{~h}$. On cooling the reaction mixture a solid appeared which was filtered and crystallized from benzene to give $\mathbf{1 6}$ as orange crystals. Yield $88 \%$, m.p. $246-248^{\circ} \mathrm{C}$. UV: $\lambda_{\max }(\varepsilon): 288.8(1.14) \mathrm{nm}$. IR $\left(\nu, \mathrm{cm}^{-1}\right): 3527-3320(\mathrm{br}$, $\mathrm{NH}, \mathrm{NH}), 1660,1608$ (C=O, CONH), 1386 (NCSN), 1255 (C-F), 1090 (C-S), 864 (aryl C-H), 658 (C-F). MS (relative intensity): 718 (M-359, $\left.-\mathrm{H}_{2} \mathrm{O}, 341,85 \%\right), 309$ (55.01), 306 (100), 290 (90.00), 209 (8.11). Anal. calcd. $\mathrm{C}_{32} \mathrm{H}_{18} \mathrm{~N}_{8} \mathrm{~S}_{2} \mathrm{O}_{4} \mathrm{~F}_{4}$ (718): C, 53.48; H, 2.50; N, 15.60; S, 8.91; F, 10.58. Found: C, 53.15; H, 2.30; N, 15.31; S, 8.56; F, 10.59. ${ }^{19}$ F NMR: $\delta-127$ and $-130 \mathrm{ppm}$.

2.1.14. 2,2,2-Tris((6-(5-fluoro-2-(4-fluorobenzamido)phenyl)5-oxo-4,5-dihydro-1,2,4-triazin-3-yl)thio)acetic acid (17). A mixture of compounds $1(0.001 \mathrm{~mol})$ and $5(0.001 \mathrm{~mol})$ in DMF $(50 \mathrm{~mL})$ was refluxed for $1 \mathrm{~h}$. The reaction mixture was left to cool at room temperature. The solid thus generated was filtered and crystallized from acetic acid to give $\mathbf{1 7}$ as yellow crystals. Yield $65 \%$, m.p. $230-232^{\circ} \mathrm{C}$. UV: $\lambda_{\max }(\varepsilon): 288.2(1.23)$ nm. IR $\left(\nu, \mathrm{cm}^{-1}\right): 3544(\mathrm{OH}), 3320(\mathrm{NH}), 2990,2898(\mathrm{C}-$ $\mathrm{H}), 1760-1610(\mathrm{C}=\mathrm{O}), 1499,1449$ (deformation $\left.\mathrm{CH}_{2}\right), 1386$ (NCSN), 1255 (C-F), 1089 (C-S), 864 (aryl C-H), 658 (C-F). ${ }^{1} \mathrm{H}$ NMR ( $\delta$, ppm): 13.72, 13.25, 12.61, 12.17 (4s, $\left.4 \mathrm{NH}\right), 10.09$, $10.06(2 \mathrm{~s}, 2 \mathrm{NH}), 7.98,7.97,7.96,7.95,7.87,7.86,7.85,7.84$, $7.83,7.54,7.40,7.39,7.38,7.35,7.10,7.09-7.05$ (aromatic protons), 5.49 (s, $1 \mathrm{H}, \mathrm{OH}) .{ }^{13} \mathrm{C}$ NMR ( $\delta$, ppm): 164.98, 163.83, 163.32 (3 C=O), 148, 140 (C-F), 132.11 (C-S), 130.23, 130.19, $130.17,129.62,129.56,129.47,129.42,125.51,125.40$ and 116.91 , $116.81,116.77,116.74,116.64,116.62,116.11,115.12,115.06,114.97$, 114.91, 77.59-77.16, 39.91-39.21 (aromatic, heteroaromatic and aliphatic carbons). Anal. calcd. $\mathrm{C}_{50} \mathrm{H}_{28} \mathrm{~N}_{12} \mathrm{~S}_{3} \mathrm{O}_{8} \mathrm{~F}_{6}$ (1134): $\mathrm{C}$, 52.55; H, 2.46; N, 14.81; S, 8.43; F, 10.05. Found: C, 51.98; H, 2.16; N, 14.53; S, 8.19; F, 9.85 .

2.1.15. 4-Fluoro-N-(4-fluoro-2-(3-((4-fluoro-2-(5-oxo-3thioxo-2,3,4,5-tetrahydro-1,2,4-triazin-6-yl)phenyl)amino)-5oxo-4,5-dihydro-1,2,4-triazin-6-yl)phenyl)benzamide (18). An equimolar mixture of $\mathbf{1}$ and $\mathbf{5}(0.001 \mathrm{~mol})$ in isopropyl alcohol $(50 \mathrm{~mL})$ was refluxed for $6 \mathrm{~h}$. Some of the solvent of reaction mixture was evaporated in vacuo. The product was separated as brownish orange solid which was crystallized from ethanol to form orange crystals of compound 18. Yield $80 \%$, m.p. $246-248^{\circ} \mathrm{C}$. UV: $\lambda_{\max }(\varepsilon): 287.8(2.07) \mathrm{nm}$. IR ( $\nu$, $\left.\mathrm{cm}^{-1}\right): 3320,3300(\mathrm{NH}, \mathrm{NH}), 1668,1620(\mathrm{C}=\mathrm{O}, \mathrm{CONH}), 1385$ (NCSN), $1255(\mathrm{C}-\mathrm{F}), 1185(\mathrm{C}=\mathrm{S}), 864(\operatorname{aryl~C}-\mathrm{H}), 657(\mathrm{C}-\mathrm{F})$. MS (relative intensity): 564 (M-196, 368, 7.0\%), 247 (0.01), 204 (3.0), 203 (5.0), 196 (1.0), 100 (1.1), 88 (100), 76 (66.0), 42 


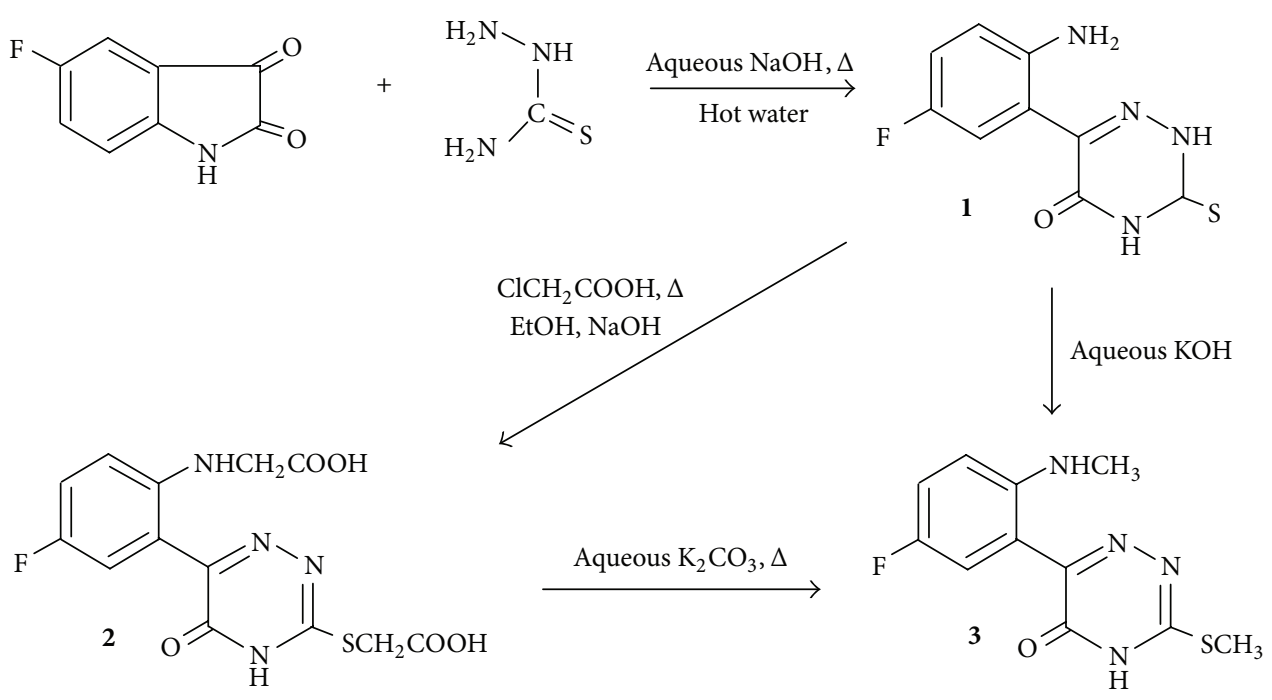

SCHEME 1

(0.5). Anal. calcd. $\mathrm{C}_{25} \mathrm{H}_{15} \mathrm{~N}_{8} \mathrm{SO}_{3} \mathrm{~F}_{3}$ (564): C, 53.34; $\mathrm{H}, 5.67$; N, 19.89; S, 5.67; F, 10.10. Found: C, 52.89; H, 5.55; N, 19.51; S, 5.38; F, 9.88. ${ }^{19}$ F NMR: $\delta-126,-128$ and -130 ppm.

\subsection{Biological Evaluation}

2.2.1. Anti-HIV-1 Activity. All the new synthesized compounds have been evaluated for their in vitro anti-HIV activity that was performed on T-4 lymphocytes infected and uninfected with HIV-1 using DMSO as solvent. The assay involves the killing of T-4 lymphocytes by HIV. Uninfected cells with the compound serve as a toxicity control, and infected and uninfected cells without the compound serve as basic controls. Cultures are incubated at $37^{\circ} \mathrm{C}$ in a $5 \%$ carbon dioxide atmosphere for 6 days. The tetrazolium salt, XTT, is added to all wells, and cultures are incubated to allow formazan color development by viable cells. Compounds that degenerate or are rapidly metabolized in the culture conditions may not show activity in this screen. Zidovudine (AZT) at $10 \mu \mathrm{M}$ was used as a control. The viability of the cells was determined spectrophotometrically to quantitate formazan production and in addition is viewed microscopically for detection of viable cells and confirmation of protective activity. Drug-treated virus-infected cells are compared with drug treated noninfected cells and with other appropriate controls (untreated infected and untreated noninfected cells, drugcontaining wells without cells) on the same plate.

2.2.2. CDK2 Inhibition Assay. CDK2-cyclin E kinase was expressed and assayed as previously described [39]. Kinase activity was expressed as a percentage of maximum activity. The concentration of the test compounds required to decrease the CDK activity by $50 \%$ was determined from dose-response curves and designated $\mathrm{IC}_{50}$.

\section{Results and Discussion}

3.1. Chemistry. The proposed synthetic strategies to obtain the target compounds are outlined in Schemes 1-5. The structures of target compounds have been established by physicochemical and spectroscopic techniques.

Reaction of compound 1 with monochloroacetic acid (1:2 by mole) in refluxing ethanolic sodium hydroxide afforded the $\alpha$-amino acid 2. Decarboxylation of 2 by warming with aqueous potassium carbonate gave 6 -[5'-fluoro-2methylaminophenyl]-3-methylthio-1,2,4-triazin-5(4H)-one 3. Compound 3 was also obtained from methylation of $\mathbf{1}$ by stirring it with methyl iodide in aqueous potassium hydroxide for $12 \mathrm{~h}$ (Scheme 1).

The IR of 1 showed strong peaks at 3528 and $3300 \mathrm{~cm}^{-1}$ for $\mathrm{NH}, \mathrm{NH}_{2}$ and at 1661 and $1255 \mathrm{~cm}^{-1}$ for $\mathrm{C}=\mathrm{O}$ and $\mathrm{C}-\mathrm{F}$ functional groups, respectively. The mass spectrum showed a peak at $m / z 254\left(\mathrm{M}+\mathrm{H}_{2} \mathrm{O}, 5 \%\right)$ with a base peak at 103 . Similarly, compound 3 exhibited in its IR spectrum three peaks at 3310, 2929, and $2862 \mathrm{~cm}^{-1}$ for $\mathrm{NH}$ and methyl groups. The mass of 3 exhibited a peak at $m / z 284\left(\mathrm{M}+\mathrm{H}_{2} \mathrm{O}, 5 \%\right)$ with a base peak at 88 . The ${ }^{1} \mathrm{H}$ NMR spectra of $\mathbf{3}$ showed two singlets at $\delta 3.2$ and $3.5 \mathrm{ppm}$ for $\mathrm{N}-\mathrm{CH}_{3}$ and $\mathrm{S}-\mathrm{CH}_{3}$ protons.

El-Gendy et al. [37], obtained 1,1-di[ $4^{\prime}$-amino- $6^{\prime}$ substituted-5-oxo-1,2,4-triazin-3-yl)thio]acetic acid as anticancer agent. Similarly refluxing compound 1 with 1,1dichloroacetic acid (2:1 by mole) in DMF produced the 1,1-di[heteroaryl]-thioacetic acid 4 through nucleophilic displacement of chlorine by thioheterocyclic moieties (Scheme 2). The UV absorption spectra of 4 recorded $\lambda_{\max }$ at $266.5 \mathrm{~nm}$ while that of $\mathbf{1}$ at $282.8 \mathrm{~nm}$.

Reaction of 1 with 4-fluorobenzoyl chloride in dry pyridine furnished 5 (Scheme 3). IR spectrum of 5 showed two carbonyl groups at 1663 and $1610 \mathrm{~cm}^{-1}$. The ${ }^{13} \mathrm{C} \mathrm{NMR}$ spectrum showed peaks at $\delta 173.15,164.14$, and $158.72 \mathrm{ppm}$ attributed to $\mathrm{C}=\mathrm{S}, \mathrm{C}=\mathrm{O}$, and $\mathrm{CONH}$ carbons. The mass 
<smiles>Nc1ccc(F)cc1-c1nnc(S)[nH]c1=O</smiles><smiles>O=C=Nc1ccc(F)cc1-c1nnc(SC(Sc2nnc(-c3cc(F)ccc3/N=C/C(=O)O)c(=O)[nH]2)C(=O)O)[nH]c1=O</smiles>

SCHEME 2

spectrum exhibited a peak at $m / z 360(\mathrm{M}+4,6 \%)$ with a base peak at 89.

Compound 5 was used as starting material for building some more $\mathrm{N}^{2}$-substituted and/or $\mathrm{C}^{3}-\mathrm{NH}$ substituted 1,2,4triazinones. Thus hydroxymethylation of 5 using methanolformaldehyde yielded 2,4-dihydroxymethyl derivative $\mathbf{6}$ which on its treatment with secondary amine as morpholine and/or 2,2-dipyridylamine under the same reaction conditions Mannich bases of the type 7 and/or 8 were isolated (Scheme 3). IR spectrum of $\mathbf{6}$ showed absorptions at 3537, $2935,2890,1498$, and $1440 \mathrm{~cm}^{-1}$ mainly attributed to $\mathrm{O}-\mathrm{H}$, $\mathrm{C}-\mathrm{H}$ stretching, and $\mathrm{CH}_{2}$ bending vibrations. The ${ }^{1} \mathrm{H}$ NMR spectrum of $\mathbf{6}$ showed peaks at $\delta 12.54,10.15$ and $8.04 \mathrm{ppm}$ for $\mathrm{NH}$ and $\mathrm{OH}$ protons. The ${ }^{13} \mathrm{C}$ NMR spectrum, however, exhibited three resonance signals at $\delta 164.99,163.32$, and $157 \mathrm{ppm}$ for $\mathrm{C}=\mathrm{S}, \mathrm{C}=\mathrm{O}$, and $\mathrm{CONH}$ groups and at $\delta 39.0 \mathrm{ppm}$ for $\mathrm{CH}_{2}$ carbon.

3-Substituted amino-1,2,4-triazine derivatives have shown chemotherapeutic [40] activities and have been proven as copper corrosion inhibitors [41]. Thus a simple nucleophilic displacement of $\mathrm{SH}$ group of 1,2,4-triazines with $\mathrm{NH}$ of different amines and sulfa drugs via reaction of compound $\mathbf{5}$ with sulfa drugs (sulfathiazole and sulfoxazoles), 4-fluoroaniline, and 2,2-dipyridylamine gave 3-substitutedamino - 6 - [ $5^{\prime}$-fluoro- $2^{\prime}$ - ( $4^{\prime \prime}$-fluorophenylcarbamidophenyl)1,2,4-triazin-5(4H)ones 9-12 (Scheme 3). The IR spectrum of compound 11 showed absorptions at 1255 and $658 \mathrm{~cm}^{-1}$ for $\mathrm{C}-\mathrm{F}$. The ${ }^{13} \mathrm{C}$ NMR spectrum showed absence of $\mathrm{C}=\mathrm{S}$ carbon. The mass spectra exhibited $m / z 437$ (M-45, 392, $1.0 \%$ ) with a base peak at 148 for the fragment $\mathrm{C}_{7} \mathrm{H}_{3} \mathrm{~N}_{3} \mathrm{~F}$.

Alkylation of 3-mercapto-1,2,4-triazinone 5 using monochloroacetic acid in dry pyridine produced the mercaptoacetic acid derivative 13. Decarboxylation of $\mathbf{1 3}$ by warming it with aqueous $\mathrm{K}_{2} \mathrm{CO}_{3}$ afforded 6-[5'-fluoro- $2^{\prime}$ ( $4^{\prime \prime}$-fluorophenyl-carbamidophenyl)-3-methylthio-1,2,4-triazin-5(4H)-one 14. Compound 14 was also obtained from treatment of compound $\mathbf{5}$ with methyl iodide in ethanolic $\mathrm{KOH}$ (Scheme 4).

Structure of $\mathbf{1 3}$ was deduced from elemental analysis and spectral data. The IR spectrum showed peaks at 3550-3100, $2919,2882,1658$, and $1610 \mathrm{~cm}^{-1}$ attributed to $\mathrm{O}-\mathrm{H}, \mathrm{N}-\mathrm{H}, \mathrm{CH}_{2}$, $\mathrm{C}=\mathrm{O}$, and $\mathrm{CONH}$ groups. In ${ }^{1} \mathrm{H}$ NMR spectrum signals at $\delta$ $14.45,12.58$, and $10.25 \mathrm{ppm}$ for $\mathrm{NH}, \mathrm{NH}$, and $\mathrm{OH}$ protons. In addition to this, a peak at $\delta 3.96$ appeared for $\mathrm{CH}_{2}$ protons. Further support was given by its ${ }^{13} \mathrm{C}$ NMR where there are three peaks for three carbonyl groups at $\delta 168.74,164.99$, and 159.27. The mass spectra recorded a peak at $\mathrm{m} / z 418$ (M-28, $390,1.00 \%$ ) with a base peak at 149 as $\mathrm{C}_{8} \mathrm{H}_{4} \mathrm{NOF}$.

Full fluorine substituted aryl-1,2,4-triazinones were synthesized by refluxing $\mathbf{1 4}$ and/or 5 with 4-fluoroaniline in ethanol led to the formation of 4 -fluoro- $\mathrm{N}$-(4-fluoro2-(3-((4-fluorophenyl)amino)-5-oxo-4,5-dihydro-1,2,4-triazin-6-yl)phenyl)benzamide one 11 (Scheme 4). However, compound 15 was also obtained by treating compound 1 with 4-fluoroaniline in boiling ethanol to give $3-\left(4^{\prime}\right.$ fluorophenyl)- 6 - ( $5^{\prime}$-fluoro - $2^{\prime}$ - aminophenyl $)$-1,2, 4- triazin$5(4 \mathrm{H})$ one 15 which upon warming with 4-fluorobenzoyl chloride in DMF yielded $\mathbf{1 1}$ (Scheme 4). The structure of $\mathbf{1 5}$ was established by elemental analysis and spectral data. The IR spectra showed absorptions at 3534, 3300, 1661, and $1640 \mathrm{~cm}^{-1}$ for $\mathrm{NH}_{2}, \mathrm{NH}$, and $\mathrm{C}=\mathrm{O}$ functional groups. The spectrum did show two peaks at 1255 and $658 \mathrm{~cm}^{-1}$ for $\mathrm{C}-\mathrm{F}$ group. The ${ }^{13} \mathrm{C}$ NMR spectrum lacks a signal for $\mathrm{C}=\mathrm{S}$ carbon.

Oxidation of compound 5 by refluxing with sulfur flowers in dry benzene yielded the disulfide $\mathbf{1 6}$ (Scheme 5). Due to higher nucleophilicity of sulfur when compared with oxygen and nitrogen, the removal of $\mathrm{H}^{+}$from $\mathrm{S}-\mathrm{H}$ is easily followed by removal of $\mathrm{H}^{-}$from other molecules to form a disulfide 16 with evolution of hydrogen (Scheme 5). The IR spectrum showed an absorption band at $1090 \mathrm{~cm}^{-1}$ for C-S-S-C group. The mass recorded $m / z 341\left(\mathrm{M}-18,-\mathrm{H}_{2} \mathrm{O}, 85 \%\right)$ with a base peak at $m / z 306$ for $\mathrm{C}_{16} \mathrm{H}_{5} \mathrm{~N}_{3} \mathrm{SOF}$. 


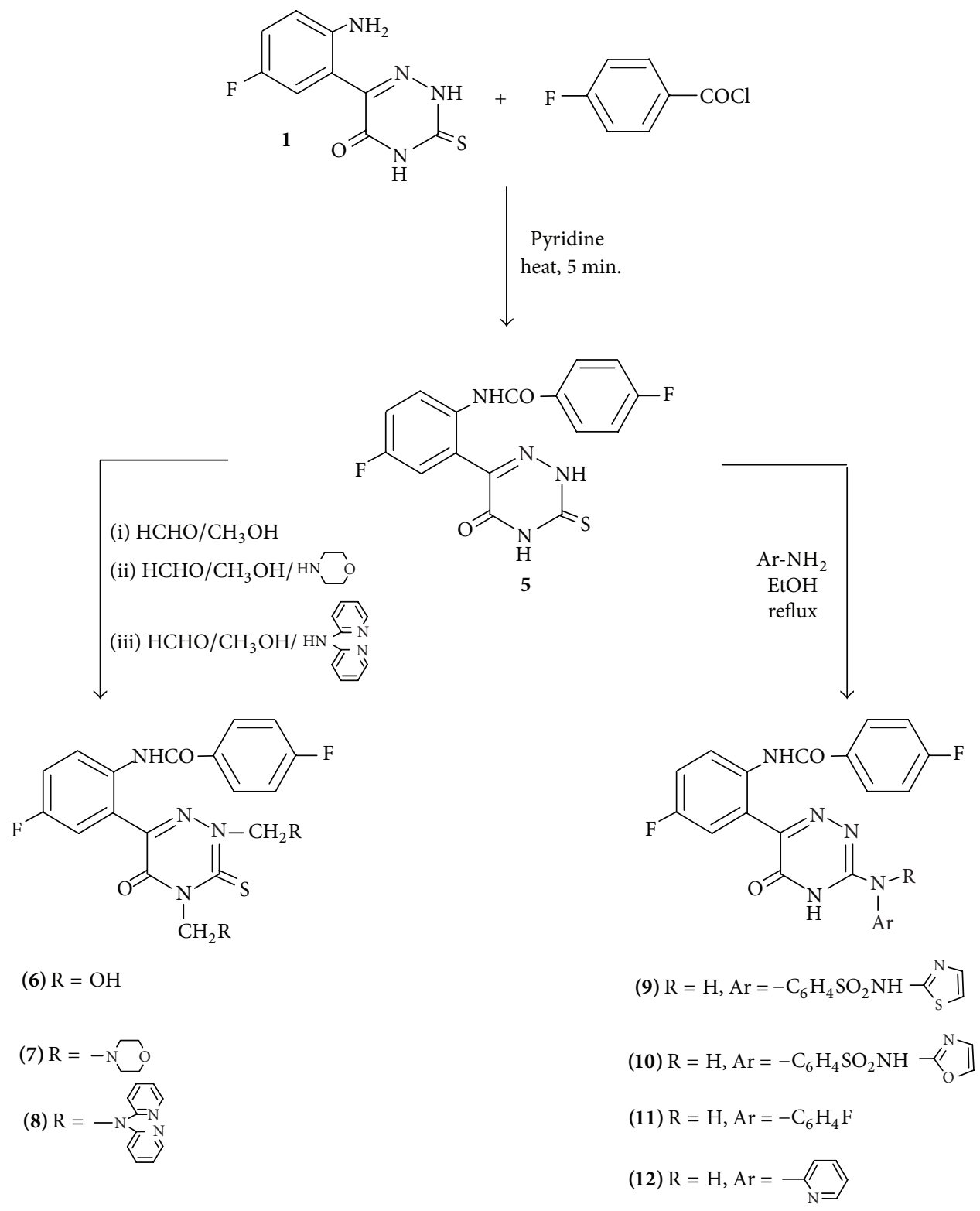

SCHEMe 3

Treatment of compound 5 with 1,1,1-trichloroacetic acid in warming DMF generates a thioether 17 (Scheme 5). The IR spectrum showed absorption bands at 1255 and $1089 \mathrm{~cm}^{-1}$ which are attributed to $\mathrm{C}-\mathrm{F}$ and $\mathrm{C}-\mathrm{S}$ of the thioether. The ${ }^{13} \mathrm{C}$ NMR spectrum lacks a signal for $\mathrm{C}=\mathrm{S}$ carbon. However, resonances at $\delta 132.11 \mathrm{ppm}$ for $\mathrm{C}-\mathrm{S}$ carbon in addition to a signal at $\delta 164.98 \mathrm{ppm}$ for carbonyl carbon.

Finally synthesis of fluorine substituted 1,2,4-triazinone bearing other amino-1,2,4-triazinone $\mathbf{1 8}$ was done by refluxing compounds $\mathbf{1}$ and $\mathbf{1 5}$ in boiling isopropyl alcohol as amination reaction (Scheme 5). The IR spectrum of $\mathbf{1 8}$ showed bands at 3320, 3300, 1668, 1620, and $1185 \mathrm{~cm}^{-1}$ for $\mathrm{NH}, \mathrm{NH}$, $\mathrm{C}=\mathrm{O}, \mathrm{CONH}$, and $\mathrm{C}=\mathrm{S}$ groups. The mass spectrum showed a peak at $\mathrm{m} / z 368(\mathrm{M}-196,7 \%)$ along with a base peak at 89 for $\mathrm{CH}_{2} \mathrm{~N}_{3} \mathrm{~S}$.
The presence of fluorine was confirmed by ${ }^{19} \mathrm{~F}$ NMR. A single fluorine atom attached to phenyl and/or benzoyl ring appeared in the region $\delta 120-130 \mathrm{ppm}$ using hexafluorobenzene as internal standard.

The mass fragmentation patterns of some of the target molecules are mentioned in Schemes 6, 7, 8, 9, and 10.

\subsection{Biological Evaluation}

3.2.1. Anti-HIV-1 Activity. The present work aimed to synthesize fluorine substituted 1,2,4-triazinones as potential anti-HIV-1 compounds as nucleoside, nonnucleoside reverse transcriptase, protease, and fusion inhibitors. The newly synthesized compounds have been evaluated for their in vitro anti-HIV activities on T4 lymphocytes, uninfected, or 
<smiles>O=C(Nc1ccc(F)cc1-c1nnc(C(=O)O)[nH]c1=O)c1ccc(F)cc1</smiles>

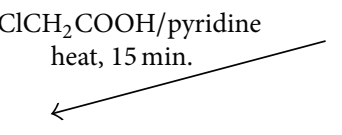

$\underset{\text { warm }}{\text { Aqueous } \mathrm{K}_{2} \mathrm{CO}_{3}} \downarrow$<smiles>CSc1nnc(-c2cc(F)ccc2NC(=O)c2ccc(F)cc2)c(=O)[nH]1</smiles>
r.t. $\mathrm{CH}_{3} \mathrm{I}$<smiles>O=C(Nc1ccc(F)cc1-c1n[nH]c(=S)[nH]c1=O)c1ccc(F)cc1</smiles><smiles>Nc1ccc(F)cc1</smiles>
$\mathrm{EtOH}$, reflux lcoholic $\mathrm{KOH}$

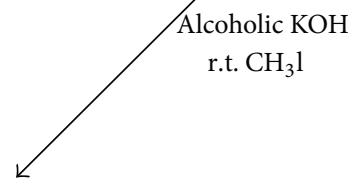<smiles>O=C(Nc1ccc(F)cc1-c1nnc(Nc2ccc(F)cc2)[nH]c1=O)c1ccc(F)cc1</smiles><smiles>O=C(Cl)c1ccc(F)cc1</smiles>
15<smiles>Nc1ccc(F)cc1-c1nnc(Nc2ccc(F)cc2)[nH]c1=O</smiles>

Scheme 4

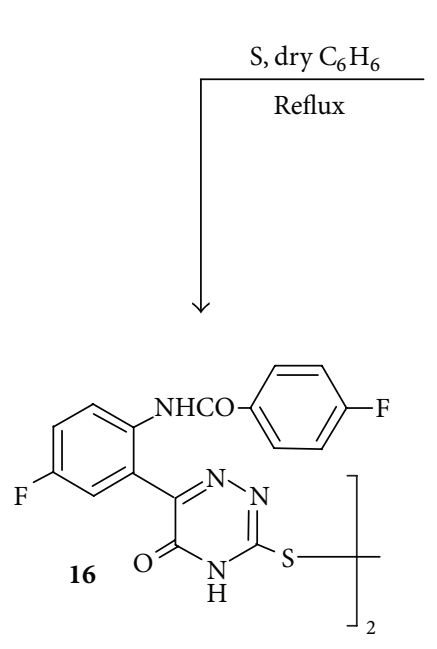<smiles>O=C(Nc1ccc(F)cc1-c1n[nH]c(=S)[nH]c1=O)c1ccc(F)cc1</smiles><smiles>Nc1ccc(F)cc1-c1n[nH]c(=S)[nH]c1=O</smiles><smiles>CCCC(C)(CC(=O)O)c1nnc(-c2cc(F)ccc2NC=O)c(=O)[nH]1</smiles><smiles>O=C(Nc1ccc(F)cc1-c1nnc(Nc2ccc(F)cc2-c2n[nH]c(=S)[nH]c2=O)[nH]c1=O)c1ccc(F)cc1</smiles>

Scheme 5

infected with HIV using DMSO as solvent. The assay basically involves the killing of T4 lymphocytes by HIV compounds that degenerate or are rapidly metabolized in the culture conditions may not show activity in this screen. The viability of the cells was determined spectrophotometrically using the tetrazolium assay procedure. The results obtained are reported in Table 1.

The results revealed by Table 1 suggest that compounds $5,11,14,16$, and 17 displayed very good anti-HIV-1 activity having a favorable selectivity index between 3 and 7 . 


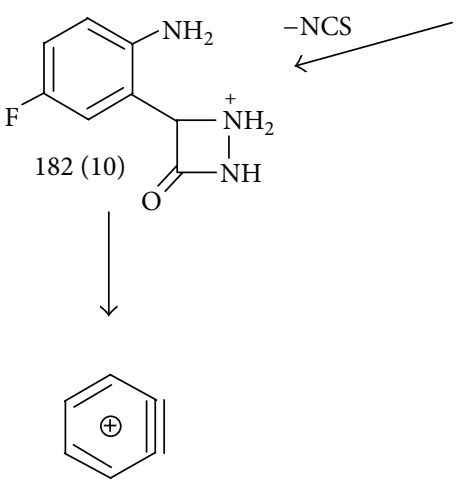

$75(35)$<smiles>CCSc1n[nH]c(=S)[nH]c1=O</smiles>

$\mathrm{M}^{+} 238\left(\mathrm{M}+\mathrm{H}_{2} \mathrm{O}, 254,5 \%\right)$<smiles>CCCCCCCCC(=O)O</smiles>

$103(100)$<smiles>Nc1ccc(F)cc1-c1nnc[nH]c1=O</smiles><smiles>[114Os]</smiles><smiles>Fc1ccc2c(c1)N2</smiles>

$108(1.1)$

SCHEME 6: Mass fragmentation pattern of compound 1.<smiles>NCO</smiles><smiles>C1CCC1</smiles>
$47(65)$<smiles>[NH3+]c1ccc(F)cc1</smiles>

$110(5.55)$<smiles>CNc1ccc(F)cc1-c1nnc(SC)[nH]c1=O</smiles>
$\mathrm{M}^{+}, 266\left(\mathrm{M}+\mathrm{H}_{2} \mathrm{O}, 284,5 \%\right)$
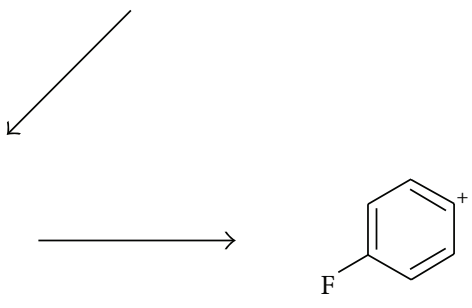

$95(1.18)$

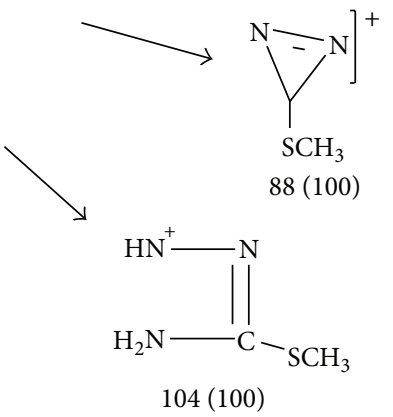

SCHEME 7: Mass fragmentation pattern of compound 3.
The $\mathrm{CC}_{50}$ and $\mathrm{EC}_{50}$ data are used to calculate the selectivity index (SI) of each compound as an estimate of a therapeutic window and a mechanism to identify candidates for efficacy studies. The other compounds though are active as antiHIV-1 agents but do not show very good selectivity index ratio. The remaining compounds exhibited an average to poor activity with selective index $<3$ (Table 1 ). In particular, a high activity level was observed for compounds $\mathbf{1 1}$ and 14. A close examination of these two compounds reveals that both the structures have 4-fluoro-N-(4-fluorophenyl)benzamide part connected at position 6 in triazinone nucleus. Similarly, in compound $\mathbf{1 6}$ which is an oxidised dimer of the active compound 5, the selectivity index is almost doubled. The antiviral activity diminishes if the triazinone ring experience crowding at carbon-3 in triazinone part as in case of $\mathbf{1 5}$ and 18. Sulfonamide moieties, however, could not contribute towards improving the antiviral profile; rather it significantly diminishes the selectivity index ratio as can be seen in compounds 9 and $\mathbf{1 0 .}$

The influence of fluorine on the acidity, hydrogen bonding, and lipophilicity of these systems can be envisaged not only a biological activity modulator but also influences the bioavailability of the drug. The active fluorine compounds obtained are capable of forming stronger DNA complexes than their nonfluorinated analogs. These results suggest that the electronic nature of the chain of 3-thioxo-1,2,4-triazines tethering an intercalator that not only influences the DNAbinding process but might also be used to tune the new DNA-drug complex. It can be implied that the fluorine substituted-3-thioxo-1,2,4-triazin-5-ones represent suitable prodrug principle leading to higher bioavailability.

The biological activity depends not only on the site of fluorination and the geometry of the conjugate carbanion 


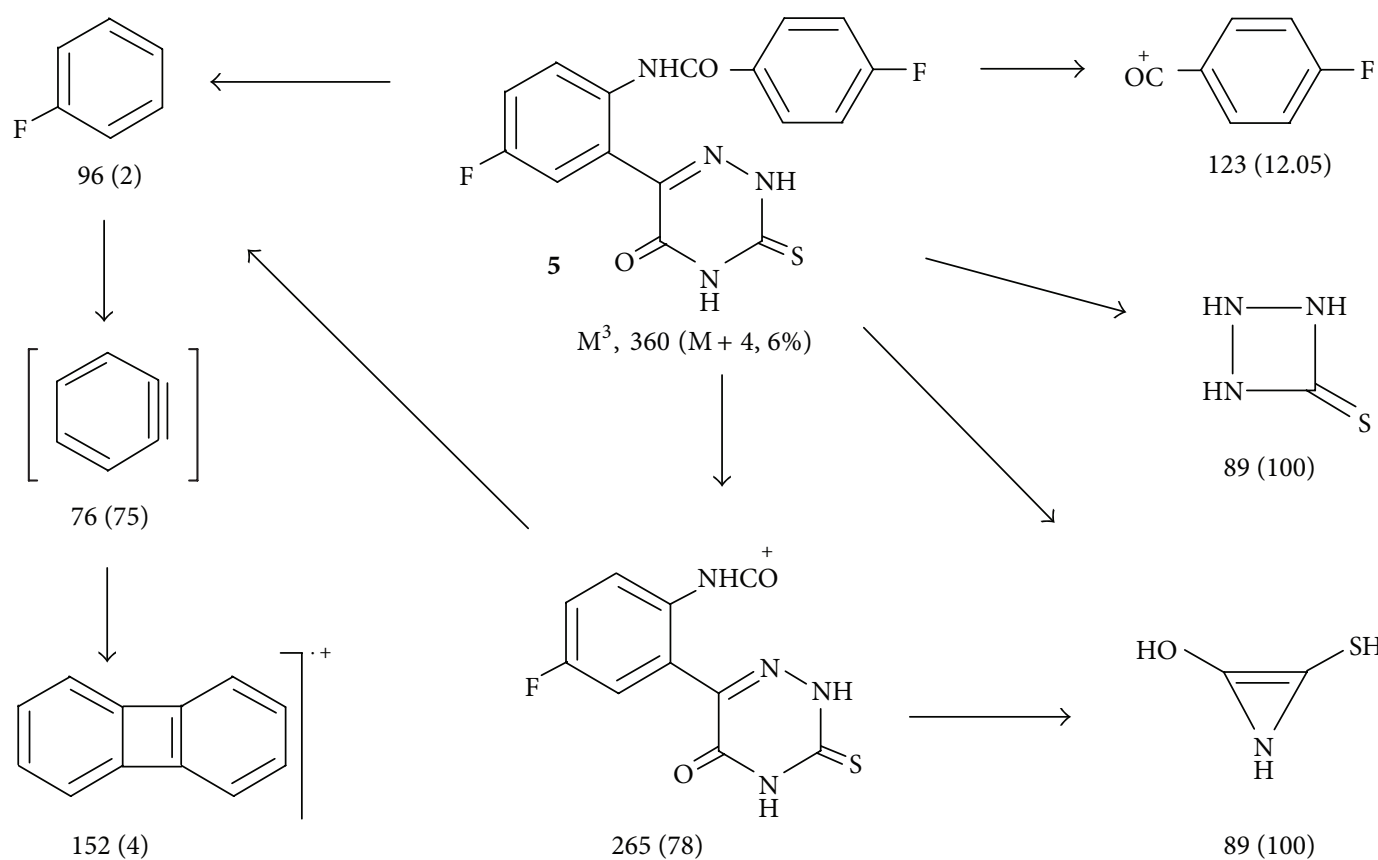

SCHEME 8: Mass fragmentation pattern of compound 5.

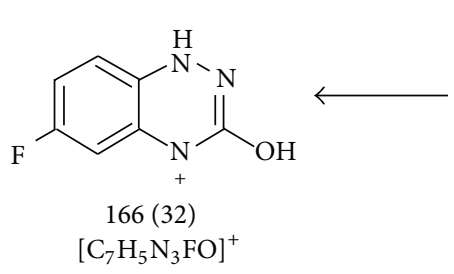<smiles>O=C(Nc1ccc(F)cc1-c1nnc(Nc2ccc(F)cc2)[nH]c1=O)c1ccc(F)cc1</smiles><smiles>CCC(C)[NH+](N)c1ccc(F)cc1</smiles>

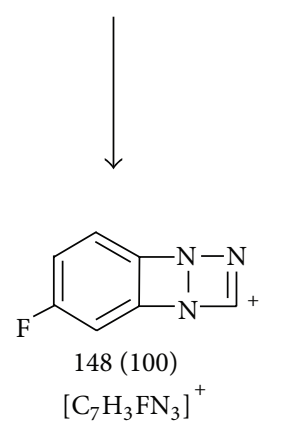<smiles>CCCC</smiles><smiles>CCCCCCC(N)=O</smiles><smiles>CNC</smiles>

SCHeme 9: Mass fragmentation pattern of compound 11.

formed but also on the total electronegativity of new heterocyclic nitrogen systems. In view of these characteristics and the results obtained in Table 1 we can infer that fluorination invariably increases $\mathrm{C}-\mathrm{H}$ acidity through a combination of inductive and hyperconjugative resonance stabilization of the carbanion and thus influences the biological activity of this series of compounds (Figure 3).
3.2.2. Anticancer Activity. The new fluorine substituted 1,2,4triazinones (1-18) have been evaluated to inhibit activity of CDK2 in a biochemical assay. The inhibitory concentration $\left(\mathrm{IC}_{50}\right)$ values were obtained according to the reported methods $[39,42]$. Olomoucine has been used as a standard. The results are reported in Table 2.

The results from Table 2 reveal that compounds 1, 3, and 14-16 show very significant CDK2 inhibitory activity. 
<smiles>CC(C)(C)Sc1nnc(-c2cc(F)ccc2NC(=O)c2ccc(F)cc2)c(=O)[nH]1</smiles>

$\mathrm{M}^{+}, 718$

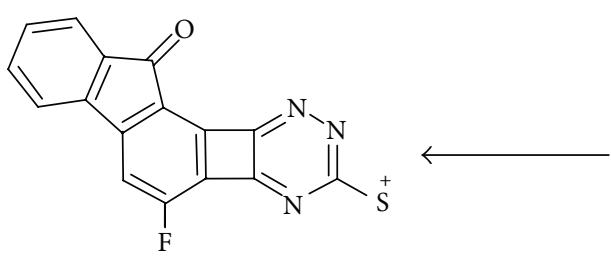

$306(100)$<smiles>O=C(Nc1ccc(F)cc1)c1nnc(S)[nH]c1=O</smiles>

359
$\mathrm{C}_{16} \mathrm{H}_{7} \mathrm{FN}_{4} \mathrm{O}$ $290(90)$

$309(55)$

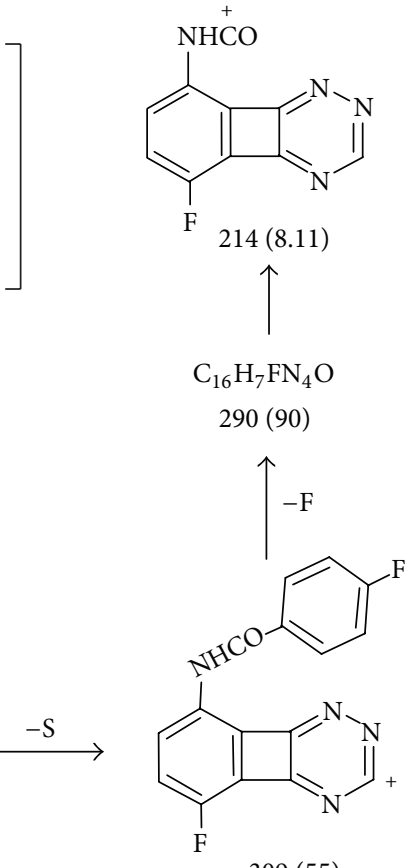

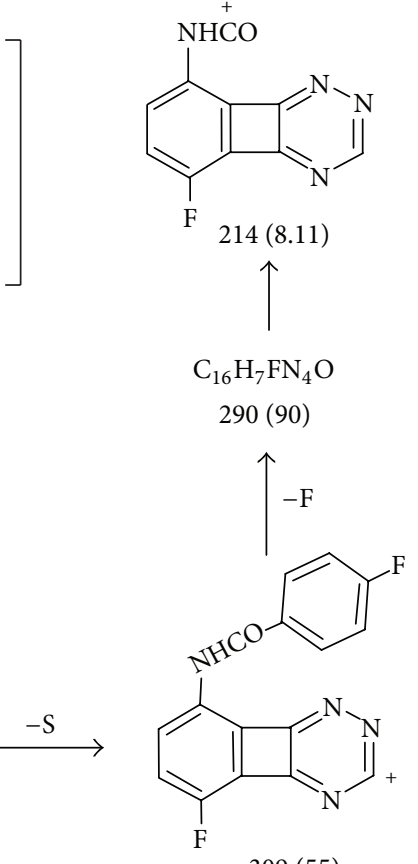<smiles>CC(O)COc1ccc(F)cc1</smiles>

$341(85)$
SCHEME 10: Mass fragmentation pattern of compound 16.
TABLE 1: Anti-HIV-1 activities of fluorinated 1,2,4-triazinones 1-18.

\begin{tabular}{lcccc}
\hline Compounds & $\mathrm{EC}_{50}(\mu \mathrm{M})^{\mathrm{a}}$ & $\mathrm{CC}_{50}(\mu \mathrm{M})^{\mathrm{b}}$ & $\mathrm{SI}^{\mathrm{c}}$ & $\log \mathrm{P}$ \\
\hline $\mathbf{1}$ & $10.8 \pm 1.0$ & $25 \pm 1.0$ & 2.51 & 0.64 \\
$\mathbf{3}$ & $20 \pm 1.0$ & $49 \pm 1.0$ & 2.50 & 2.09 \\
$\mathbf{5}$ & $25 \pm 1.0$ & $75 \pm 1.0$ & 3.00 & 2.4 \\
$\mathbf{6}$ & $30 \pm 1.0$ & $60 \pm 1.0$ & 2.00 & 2.39 \\
$\mathbf{9}$ & $35 \pm 1.0$ & $75 \pm 1.0$ & 2.20 & 4.41 \\
$\mathbf{1 0}$ & $42 \pm 1.0$ & $82 \pm 1.0$ & 2.11 & 3.04 \\
$\mathbf{1 1}$ & $6.6 \pm 1.0$ & $44 \pm 2.0$ & 7.15 & 4.44 \\
$\mathbf{1 4}$ & $5.8 \pm 1.0$ & $35 \pm 1.0$ & 6.2 & 3.56 \\
$\mathbf{1 5}$ & $14.0 \pm 1.0$ & $29 \pm 1.0$ & 2.01 & 2.67 \\
$\mathbf{1 6}$ & $6.9 \pm 1.0$ & $36 \pm 1.1$ & 5.1 & 7.07 \\
$\mathbf{1 7}$ & $25 \pm 1.8$ & $76 \pm 1.0$ & 3.01 & - \\
$\mathbf{1 8}$ & $50 \pm 1.1$ & $90 \pm 2.1$ & 1.8 & 3.69 \\
\hline
\end{tabular}

${ }^{\mathrm{a}} \mathrm{EC}_{50}$ : inhibiting $\mathrm{p} 24$ production in the MT- 4 cells.

${ }^{\mathrm{b}} \mathrm{CC}_{50}$ : concentration of compound that reduces the viability of mockinfected cells by $50 \%$, as determined by the MTT method.

${ }^{\mathrm{c}} \mathrm{SI}$ : selectivity index; $\mathrm{CC}_{50} / \mathrm{EC}_{50}$.

Compound 3 was found to be as active as olomoucine, while compounds $\mathbf{1}$ and $\mathbf{1 4 - 1 6}$ were more potent than the standard compound. Once again the same structural features are present in active compounds as is evident in compounds active as anti-HIV-1.

\section{Conclusions}

According to the data obtained from the biological assay five compounds $5,11,14,16$, and 17 have shown very good

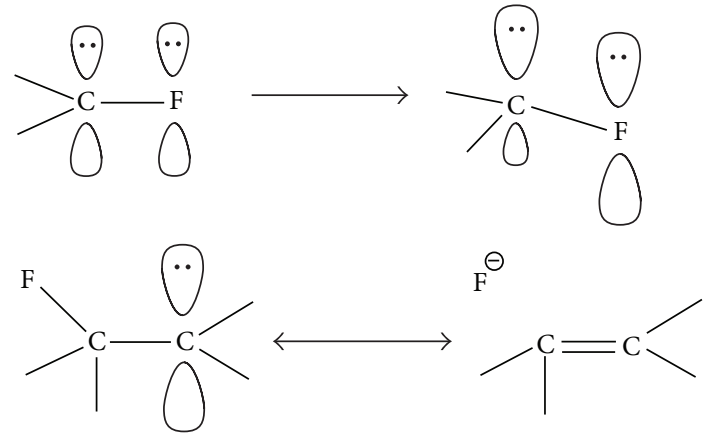

FIGURE 3: Hyperconjugation between atom and C-H.

anti-HIV activity in MT-2 cells. Similarly, five compounds $(\mathbf{1}, \mathbf{3}$, and 14-16) have exhibited remarkable CDK2 inhibition activity. Compounds 14 and 16 can be considered as a significant matrix for the design and synthesis of novel candidates with dual anti-HIV and anticancer activities. Further investigation into the other aspects of structure activity relationship studies of this series of compounds is required in order to explore the scope and limitation of its biological activities.

\section{Conflict of Interests}

The authors declare that there is no conflict of interests regarding the publication of this paper. 
TABLE 2: CDK2 inhibiting activity of fluorinated 1,2,4-triazinones 118.

\begin{tabular}{lc}
\hline Compounds & $\mathrm{IC}_{50} \pm \mathrm{SD}(\mu \mathrm{M})^{\mathrm{a}}$ \\
\hline $\mathbf{1}$ & $3.8 \pm 1.7$ \\
$\mathbf{3}$ & $5.0 \pm 1.7$ \\
$\mathbf{5}$ & $12.8 \pm 1.7$ \\
$\mathbf{6}$ & $12.2 \pm 1.7$ \\
$\mathbf{9}$ & $14.0 \pm 1.5$ \\
$\mathbf{1 0}$ & $15.0 \pm 2.5$ \\
$\mathbf{1 1}$ & $15.0 \pm 5.0$ \\
$\mathbf{1 4}$ & $4.5 \pm 2.8$ \\
$\mathbf{1 5}$ & $4.1 \pm 2.8$ \\
$\mathbf{1 6}$ & $4.0 \pm 2.8$ \\
$\mathbf{1 7}$ & $16.2 \pm 5.0$ \\
$\mathbf{1 8}$ & $18.1 \pm 4.0$ \\
Olomoucine & $5.0 \pm 1.0$ \\
\hline${ }^{\mathrm{a}} \mathrm{IC}_{50}$ in $\mu \mathrm{M} / d_{m}{ }^{-1}$. & \\
SD: standard deviation. &
\end{tabular}

\section{Acknowledgments}

The project was funded by the Deanship of Scientific Research (DSR), King Abdulaziz University, Jeddah, under Grant no. 491/130/1433. The authors therefore acknowledge with thanks DSR technical and financial support.

\section{References}

[1] E. De Clercq, "New developments in anti-HIV chemotherapy," Biochimica et Biophysica Acta, Molecular Basis of Disease, vol. 1587, no. 2-3, pp. 258-275, 2002.

[2] E. De Clercq, "Antiviral drugs in current clinical use," Journal of Clinical Virology, vol. 30, no. 2, pp. 115-133, 2004.

[3] C. M. Kitchen, S. G. Kitchen, J. A. Dubin, and M. S. Gottlieb, "Initial virological and immunologic response to highly active antiretroviral therapy predicts long-term clinical outcome," Clinical Infectious Diseases, vol. 33, no. 4, pp. 466-472, 2001.

[4] W. M. Valenti, "HAART is cost-effective and improves outcomes," AIDS Reader, vol. 11, no. 5, pp. 260-262, 2001.

[5] J. T. King Jr., A. C. Justice, M. S. Roberts, C.-C. H. Chang, and J. S. Fusco, "Long-term HIV/AIDS survival estimation in the highly active antiretroviral therapy era," Medical Decision Making, vol. 23, no. 1, pp. 9-20, 2003.

[6] A. Carr, "Toxicity of antiretroviral therapy and implications for drug development," Nature Reviews Drug Discovery, vol. 2, no. 8, pp. 624-634, 2003.

[7] J. Martinez-Picado, M. P. DePasquale, N. Kartsonis et al., "Antiretroviral resistance during successful therapy of HIV type 1 infection," Proceedings of the National Academy of Sciences of the United States of America, vol. 97, no. 20, pp. 10948-10953, 2000.

[8] S. J. Little, S. Holte, J.-P. Routy et al., "Antiretroviral-drug resistance among patients recently infected with HIV," The New England Journal of Medicine, vol. 347, no. 6, pp. 385-394, 2002.

[9] E. Pomarnacka and A. Kornicka, "Synthesis and in vitro anticancer and anti-HIV evaluation of new 2-mercaptobenzenesulfonamides," Farmaco, vol. 56, no. 8, pp. 571-577, 2001.
[10] D. A. Karnofsky, "Mechnaism of action of anticancer drugs at a cellular level," Ca-A Cancer Journal for Clinicians, vol. 18, no. 4, pp. 232-234, 1968.

[11] S. T. M. Makki, D. A. Bakhotmah, R. M. Abdel-Rahman, and M. S. El-Shahawy, "Designing and synthesis of new fluorine substituted pyrimidine-thion-5-carbonitriles and the related derivatives as photochemical probe agents for inhibition of vitiligo disease," International Journal of Organic Chemistry, vol. 2, no. 3, pp. 311-320, 2012.

[12] R. M. Abdel-Rahman, M. S. I. T. Makki, and W. A. Bawazir, "Synthesis of some more fluorine heterocyclic nitrogen systems derived from sulfa drugs as photochemical probe agents for inhibition of vitiligo disease-part i," E-Journal of Chemistry, vol. 8, no. 1, pp. 405-414, 2011.

[13] R. M. Abdel-Rahman, M. S. I. T. Makki, and W. A. B. Bawazir, "Synthesis of fluorine heterocyclic nitrogen systems derived from sulfa drugs as photochemical probe agents for inhibition of vitiligo disease-Part II," E-Journal of Chemistry, vol. 7, no. 1, pp. S93-S102, 2010.

[14] D.-J. Wang, L. Fan, C.-Y. Zheng, and Z.-D. Fang, "Synthesis and anti-microbial activity of some new fluorinated $1 \mathrm{H}$-pyrazoles," Journal of Fluorine Chemistry, vol. 131, no. 5, pp. 584-586, 2010.

[15] R. Filler and R. Saha, "Fluorine in medicinal chemistry: a century of progress and a 60-year retrospective of selected highlights," Future Medicinal Chemistry, vol. 1, no. 5, pp. 777791, 2009.

[16] G. Sandford, "Elemental fluorine in organic chemistry (19972006)," Journal of Fluorine Chemistry, vol. 128, no. 2, pp. 90-104, 2007.

[17] C. Isanbor and D. O'Hagan, "Fluorine in medicinal chemistry: a review of anti-cancer agents," Journal of Fluorine Chemistry, vol. 127, no. 3, pp. 303-319, 2006.

[18] B. E. Smart, "Fluorine substituent effects (on bioactivity)," Journal of Fluorine Chemistry, vol. 109, no. 1, pp. 3-11, 2001.

[19] S. Liu, X. Qian, G. Song, J. Chen, and W. Chen, "Fluorine containing heterocyclic compounds: Synthesis of 6-substituted2-substituted-aryl-1,2,4-triazolo[5,1-b] 1,3,5-thiadiazin-7-one derivatives," Journal of Fluorine Chemistry, vol. 105, no. 1, pp.111115,2000

[20] R. M. Abdel-Rahman, "Synthesis and chemistry of fluorine containing bioactive 1,2,4-triazines-an overview: chemistry of uncondensed 1,2,4-triazines, part III," Pharmazie, vol. 54, no. 11, pp. 791-803, 1999.

[21] T. . Ramadan, R. M. Abdel-Rahman, and M. Seada, "Studies on complexes of $\mathrm{Cu}(\mathrm{II}), \mathrm{Ni}(\mathrm{II}), \mathrm{Co}(\mathrm{II})$ and $\mathrm{Ln}(\mathrm{II})$ with $3-(\alpha$ benzoyl)benzylidene hydrazino-5,6-diphenyl-1,2,4-triazine," Asian Journal of Chemistry, vol. 4, pp. 569-575, 1992.

[22] A. A. T. Ramadan, R. M. Abdel-Rahman, M. A. El-Behairy, A. I. Ismail, and M. M. Mahmoud, "The thermodynamics of complexation of transition and lanthanide ions by 3-( $\alpha$ carboxymethylaminobenzyli-denehydrazino)-5,6-diphenyl-1, 2,4 triazine (HipHt)," Thermochimica Acta, vol. 222, no. 2, pp. 291-303, 1993.

[23] F. M. Foss, "Nucleoside analogs and antimetabolite therapies for myelodysplastic syndrome," Best Practice and Research: Clinical Haematology, vol. 17, no. 4, pp. 573-584, 2004.

[24] G. S. Cockerill and K. E. Lackey, "Small molecule inhibitors of the class 1 receptor tyrosine kinase family," Current topics in medicinal chemistry, vol. 2, no. 9, pp. 1001-1010, 2002.

[25] E. K. Rowinsky, "Signal Events: cell Signal Transduction and Its Inhibition in Cancer," Oncologist, vol. 8, no. 3, pp. 5-17, 2003. 
[26] V. Aparna, G. Rambabu, S. K. Panigrahi, J. A. R. P. Sarma, and G. R. Desiraju, "Virtual screening of 4-anilinoquinazoline analogues as EGFR kinase inhibitors: importance of hydrogen bonds in the evaluation of poses and scoring functions," Journal of Chemical Information and Modeling, vol. 45, no. 3, pp. 725738, 2005.

[27] S. Emanuel, R. H. Gruninger, A. Fuentes-Pesquera et al., "A vascular endothelial growth factor receptor-2 kinase inhibitor potentiates the activity of the conventional chemotherapeutic agents paclitaxel and doxorubicin in tumor xenograft models," Molecular Pharmacology, vol. 66, no. 3, pp. 635-647, 2004.

[28] R. M. Abdel-Rahman, "Role of uncondensed 1,2,4-triazine compounds and related heterobicyclic systems as therapeutic agents-a review," Pharmazie, vol. 56, no. 1, pp. 18-22, 2001.

[29] R. M. Abdel-Rahman, "Synthesis and anti human immune virus activity of some new fluorine containing substituted-3-thioxo1,2,4-triazin-5-ones," Farmaco, vol. 46, no. 2, pp. 379-389, 1991.

[30] R. M. Abdel-Rahman, "Synthesis of some new fluorine bearing trisubstituted 3-thioxo-1,2,4- triazin-5-ones as potential anticancer agents," Farmaco, vol. 47, no. 3, pp. 319-326, 1992.

[31] R. M. Abdel-Rahman, M. Seada, M. Fawzy, and I. El-Baz, "Synthesis of some new thioethers of 1,2,4-triazine-3-hydrazones and assays for their anticancer and anti human immune virus activities," Farmaco, vol. 48, no. 3, pp. 397-406, 1993.

[32] R. M. Abdel-Rahman, M. Seada, M. Fawzy, and I. El-Baz, "Synthesis of some new 1,6-dihydro-3-substituted 6-spiro-(9'fluorene)-1,2,4-triazin-5-(4H)-ones as potential anti HIV and anticancer drugs," Pharmazie, vol. 49, no. 10, pp. 729-733, 1994.

[33] R. M. Abdel-Rahman, M. Seada, M. Fawzy, and I. El-Baz, "Synthesis of some new heterobicyclic compounds containing spiro1,2,4-triazine moiety as potential anti-HIV and anticancer agents," Pharmazie, vol. 49, no. 11, pp. 811-814, 1994.

[34] A. M. Abdel-Halim, Z. El-Gendy, and R. M. Abdel-Rahman, "Synthesis and biological activity of 1,2,4-triazinotriazinone derivatives," Pharmazie, vol. 50, no. 11, pp. 726-729, 1995.

[35] R. M. Abdel-Rahman, J. M. Morsy, F. Hanafy, and H. A. Amene, "Synthesis of heterobicyclic nitrogen systems bearing the 1,2,4triazine moiety as anti-HIV and anticancer drugs: part I," Pharmazie, vol. 54, no. 5, pp. 347-351, 1999.

[36] R. M. Abdel-Rahman, J. M. Morsy, S. El-Edfawy, and H. A. Amine, "Synthesis of some new heterobicyclic nitrogen systems bearing the 1,2,4- triazine moiety as anti-HIV and anti-cancer drugs, part II," Pharmazie, vol. 54, no. 9, pp. 667-671, 1999.

[37] Z. El-Gendy, J. M. Morsy, H. A. Allimony, W. R. Abdel-Monem Ali, and R. M. Abdel-Rahman, "Synthesis of heterobicyclic nitrogen systems bearing the 1,2,4-triazine moiety as anti-HIV and anticancer drugs, part III," Pharmazie, vol. 56, no. 5, pp. 376-383, 2001.

[38] Z. El-Gendy, J. M. Morsy, H. A. Allimony, W. R. Abdel-Monem, and R. M. Abdel-Rahman, "Synthesis of heterobicyclic nitrogen systems bearing a 1,2,4-triazine moiety as anticancer drugs: part IV," Phosphorus, Sulfur and Silicon and the Related Elements, vol. 178, no. 9, pp. 2055-2071, 2003.

[39] V. Kryštof, P. Cankař, I. Fryšová et al., “4-Arylazo-3,5-diamino$1 H$-pyrazole CDK inhibitors: SAR study, crystal structure in complex with CDK2, selectivity, and cellular effects," Journal of Medicinal Chemistry, vol. 49, no. 22, pp. 6500-6509, 2006.

[40] R. M. Abdel-Rahman, "Chemistry of uncondensed 1,2,4triazines, part iv synthesis and chemistry of bioactive 3-amino1,2,4-triazines and related compounds-an overview," Pharmazie, vol. 56, no. 4, pp. 275-286, 2001.
[41] K. F. Khaled, "Adsorption and inhibitive properties of a new synthesized guanidine derivative on corrosion of copper in 0.5 $\mathrm{M} \mathrm{H}_{2} \mathrm{SO}_{4}$," Applied Surface Science, vol. 255, no. 5, pp. 1811-1818, 2008.

[42] T. Gucký, E. Řezníčková, P. Džubák, M. Hajdúch, and V. Kryštof, "Synthesis and anticancer activity of some 1,5-diaryl-3-(3,4,5Džubák trihydroxyphenyl)-1H-pyrazolo[4,3-e][1,2,4] triazines," Monatshefte fur Chemie, vol. 141, no. 6, pp. 709-714, 2010. 

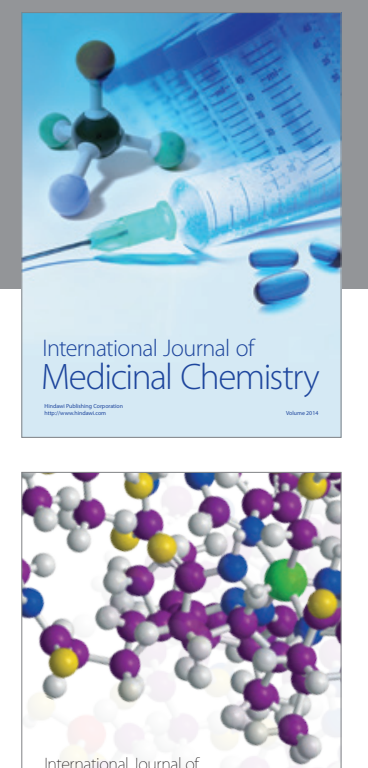

\section{Carbohydrate} Chemistry

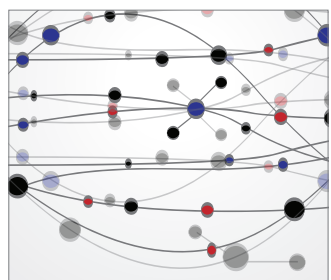

The Scientific World Journal
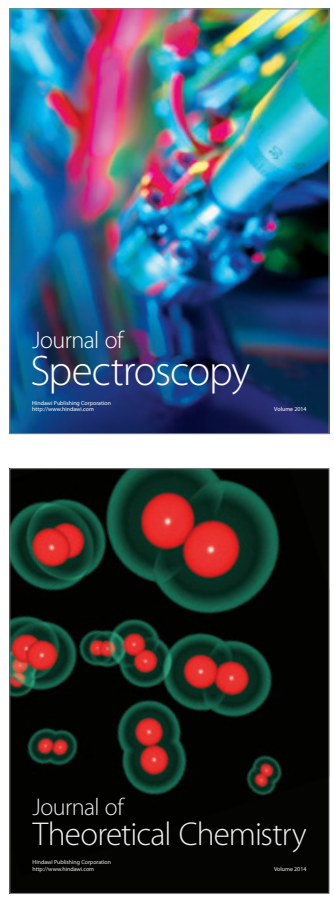
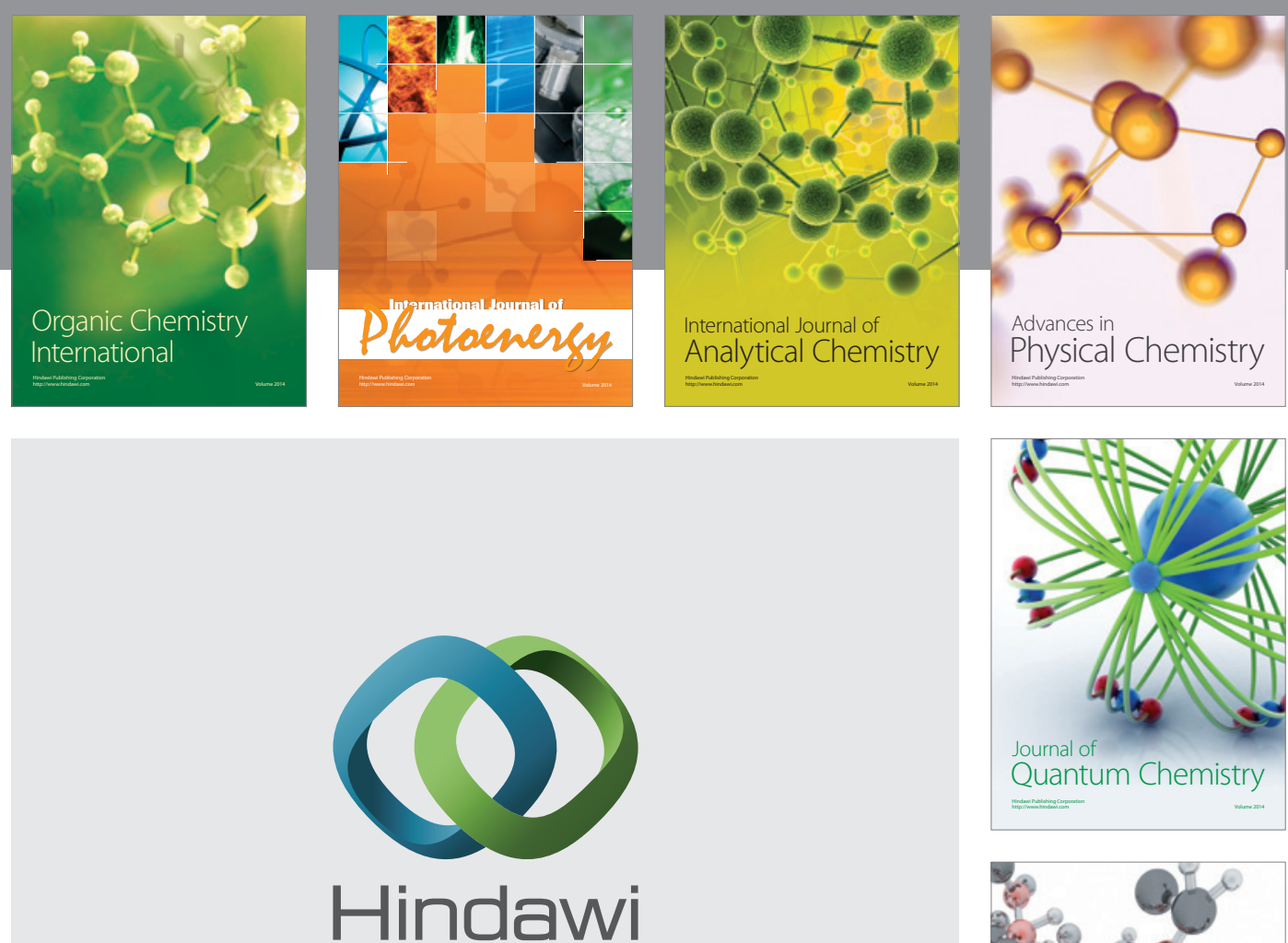

Submit your manuscripts at

http://www.hindawi.com

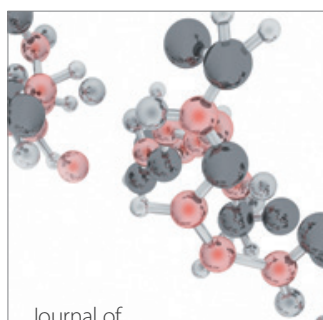

Analytical Methods

in Chemistry

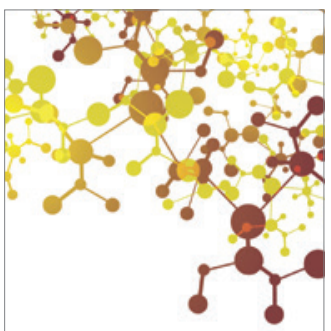

Journal of

Applied Chemistry

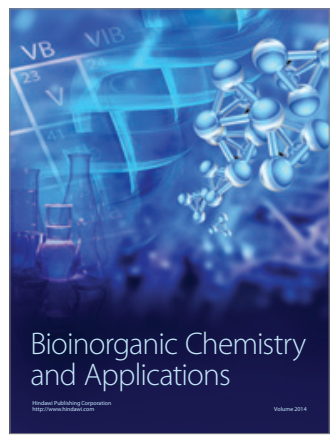

Inorganic Chemistry
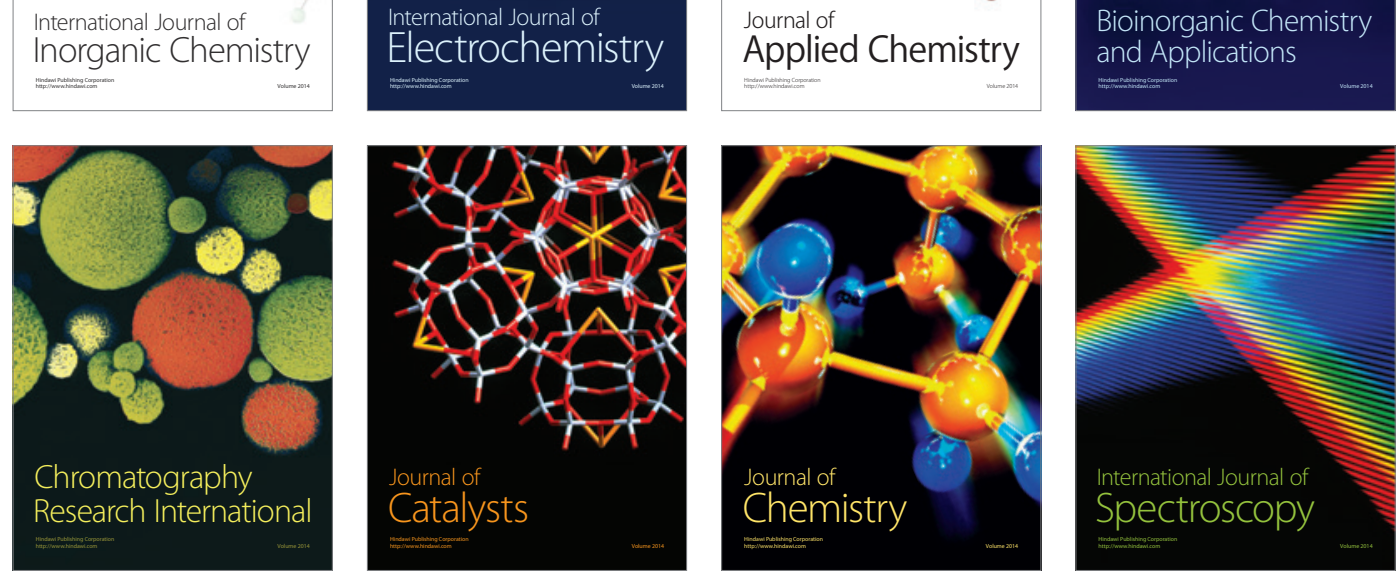